\title{
Nuevo Patrón arquitectónico ParaCas en LuCANAS, SIERRA SUR DEL PERÚ
}

\author{
Markus Reindel a y Johny Isla ${ }^{b}$
}

\begin{abstract}
Resumen
Trabajos de prospección arqueológica realizados en la parte alta de los valles de Palpa, en la vertiente occidental de los Andes, nos han permitido identificar y registrar un importante número de asentamientos pertenecientes a la cultura Paracas, de manera especial, aquellos pertenecientes a las épocas Paracas Medio (550 a 350 a.C.) y Paracas Tardio (350 a 200 a.C.), entre los que destacan varios sitios bastante grandes que presentan construcciones de piedra que conforman estructuras arquitectónicas en forma de flor. Se trata de construcciones en donde se pueden distinguir patios hundidos de forma de media luna o de forma circular, alrededor de los cuales se disponen recintos en forma de "D" que presentan pequeñas terrazas delante del muro recto.

Recientes excavaciones en área realizadas en Cutamalla, uno de los asentamientos más grandes y mejor conservados de la zona, nos han permitido documentar con detalle este tipo de estructuras arquitectónicas, y conocer sus rasgos formales y constructivos, su filiación cultural, asi como las actividades que se realizaban en ellas.

Todos los datos indican que las estructuras arquitectónicas en forma de flor constituyen un nuevo patrón arquitectónico Paracas Tardio, cuya distribución se limita a una parte de la sierra de Lucanas, Ayacucho, y que, hasta el momento, viene a ser único en toda la sierra sur del Perú.
\end{abstract}

Palabras clave: valles de Palpa, cultura Paracas, Ocucaje 9, Cutamalla, estructuras arquitectónicas en forma de flor

\section{Abstract}

\section{NEW PARACAS ARCHITECTURE PATTERN IN LUCANAS, SOUTHERN HIGHLANDS PERU}

During our archaeological surveys in the upper reaches of the Palpa valleys, at the western slope of the Andes, we identified and registered a large number of settlements of the Paracas culture, most of them dating to the Middle Paracas (550-350 BC) and Late Paracas (350-200 BC) period. Of special interest are several large sites with stone buildings with circular ground plans, similar to the shape of a flower. The center of these architectural complexes consists of a circular or crescent shaped sunken patio, around which are arranged D-shaped enclosures with small terraces in front of the straight wall.

Through our large scale excavations in Cutamalla, one of the largest and best preserved settlements in the area, we were able to document in detail this new type of architecture and to define its formal and constructive features, as well as its cultural affliation and the activities carried out within the structures.

All the data recovered so far indicate that these circular structures have to be considered as a new architectural type of the Late Paracas period. The distribution of this new architectural pattern is limited to a part of the highlands of Lucanas, Ayacucho, and, as far as we know, seems to be unique in the southern highlands of Peru.

Keywords: Palpa valleys, Paracas culture, Ocucaje 9, Cutamalla, circular estructures

${ }^{a}$ Instituto Arqueológico Alemán (DAI), Comisión de Arqueología para Culturas Extraeuropeas (KAAK), Bonn Correo electrónico: markus.reindel@dainst.de

b Andes Centro de Investigación para la Arqueología y el Desarrollo, Lima

Correo electrónico: isla.nasca@gmail.com 


\section{Introducción}

La arquitectura monumental se considera como un rasgo típico de las culturas formativas del área central andina. Se debe indicar, sin embargo, que, durante el Período Formativo, en la costa sur del Perú, se han desarrollado sociedades complejas con una tradición artística muy sofisticada, como aquella de la cultura Paracas, pero que no tuvieron su expresión en una arquitectura de grandes volúmenes, salvo algunas huacas del valle de Chincha. Por lo tanto, surge la pregunta: ¿Cuáles son las características específicas de la arquitectura del Período Formativo en el sur del Perú y por qué no se han desarrollado en esa región centros monumentales como aquellos del centro y el norte del Perú? $\mathrm{Al}$ formular esa pregunta, nos damos cuenta de que nuestros conocimientos sobre la arquitectura y los patrones de asentamiento de las sociedades formativas del sur del Perú todavía son bastante limitados.

En nuestras recientes investigaciones realizadas en la parte alta de los valles de Palpa, en la vertiente occidental de los Andes, perteneciente a la provincia de Lucanas, Ayacucho, hemos encontrado un nuevo modelo arquitectónico relacionado con la última fase de desarrollo de la cultura Paracas, cuyo elemento básico está constituido por un recinto en forma de «D», que se presenta en número de dos, tres o más alrededor de un patio central o de un espacio abierto. En el caso ideal, se forma un círculo casi perfecto que generalmente se encuentra sobre una colina artificialmente modificada, en donde los recintos en «D» están dispuestos alrededor de un patio hundido de forma circular. En el sitio de Cutamalla, se han identificado doce conjuntos con estas características, los cuales se encuentran bastante bien conservados. Asimismo, durante los trabajos de prospección en la sierra de Lucanas, se han identificado otros seis sitios más con construcciones similares, de tal manera que se puede afirmar que se trata de un modelo arquitectónico recurrente y bien establecido de la arquitectura paracas tardío en la sierra de Lucanas.

Por el tamańo de sus componentes y su ordenamiento formal y repetitivo, este nuevo patrón arquitectónico de la cultura Paracas se puede considerar como monumental, en la medida que presenta sólidos muros, hechos con grandes piedras y que a su vez ocupan grandes espacios. Las evidencias encontradas en las excavaciones indican que se trataba de estructuras arquitectónicas en donde se realizaban actividades productivas, como la fabricación de cerámica; tallado de puntas de obsidiana; y, en especial, el acopio y transformación de lana de camélidos.

En el estado actual de las investigaciones, podemos indicar que, en la sierra sur, se ha registrado y documentado un nuevo modelo arquitectónico relacionado con la época tardía de la cultura Paracas que, hasta el momento, parece ser exclusivo de la parte alta de los valles de Palpa, en zona de Lucanas. Existe algún vínculo con tradiciones arquitectónicas de la sierra sur del Perú y del norte de Bolivia, de las cuales, sin embargo, nos faltan todavía datos concretos para poder establecer comparaciones de mayor alcance.

\section{Geografía y paisaje}

Los valles de Palpa se localizan en la parte norte de la cuenca del Río Grande, en la costa sur del Perú, en la región de Ica, pero sus nacientes o cabeceras se encuentran en la parte alta de la vertiente occidental de los Andes, y ocupan una buena parte de la sección oeste de la provincia de Lucanas, en la región de Ayacucho (Fig. 1). En este contexto, los valles de Palpa conforman un eje de integración costa-sierra único por el que se puede transitar en muy poco tiempo.

La zona de investigación se localiza en la parte alta de los valles de Palpa y Viscas y ocupa un área de más de 700 kilómetros cuadrados que se encuentra físicamente integrada por los ríos Llauta, Laramate y Ocaña (Fig. 2). Esta zona, conocida también como «Las Cabezadas», va desde los 2000 hasta los 4350 metros sobre el nivel del mar. El área central de los estudios del Proyecto Arqueológico Palpa-Lucanas se encuentra en el distrito de Laramate, donde el río del mismo nombre conforma el eje principal de la zona de investigación, mientras que los límites por el norte y sur, respectivamente, se encuentran alrededor de los poblados de Llauta y Ocaña. ${ }^{1}$ 


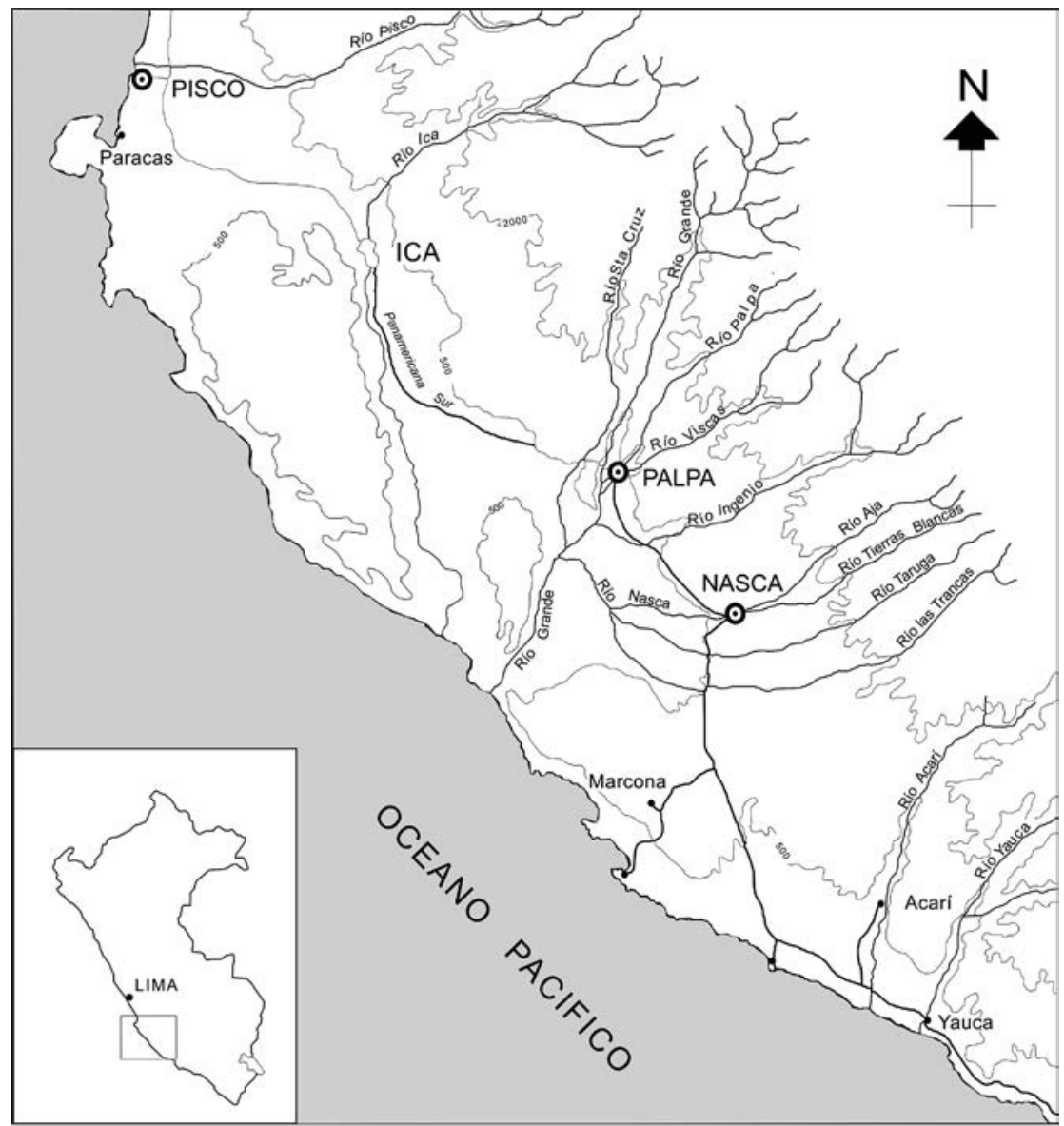

Figura 1. Mapa de costa sur del Perú con ubicación de los valles de Palpa en la parte norte de la cuenca del río Grande, cuyas nacientes se encuentran en la parte alta de la vertiente occidental de los Andes (mapa elaborado por los autores).

En general, la zona presenta una geografía bastante accidentada, que se caracteriza por la presencia de cerros altos con pendientes muy inclinadas, con crestas y farallones rocosos que se alternan con lomas, colinas y espolones de contornos suaves. Los espacios aptos para el desarrollo de la agricultura son escasos, y, por lo general, se concentran en la parte baja de los cerros, en las quebradas, $\mathrm{y}$ en bordes de los ríos principales y sus tributarios. Al igual que antes, esto es especialmente notable cerca de donde actualmente se encuentran los poblados más grandes, como Llauta, Laramate y Ocańa. Por otro lado, la amplia llanura alargada que se encuentra entre los ríos Llauta y Laramate, así como las lomas y cerros que se encuentran arriba de los 3000 metros sobre el nivel del mar, conforman espacios que estacionalmente se cubren de pastos apropiados para el desarrollo de la ganadería.

La zona de "Las Cabezadas", donde se concentra la mayor parte de las actividades humanas, se encuentra entre los 2000 y 3500 metros de altura. Esta zona presenta características especiales para la ocupación humana: clima frío y seco pero agradable; agua durante todo el año; numerosos abanicos, quebradas y lomas con fértiles campos de cultivo; y grandes lomas, laderas y pampas cubiertas de vegetación estacional aptas para el pastoreo. ${ }^{2}$ Debajo de los 2000 metros de altura se encuentra la yunga desértica, la cual, si bien presenta condiciones climáticas agradables para la vida, es bastante seca y agreste, y los lugares aptos para la ocupación humana se restringen a los pocos 


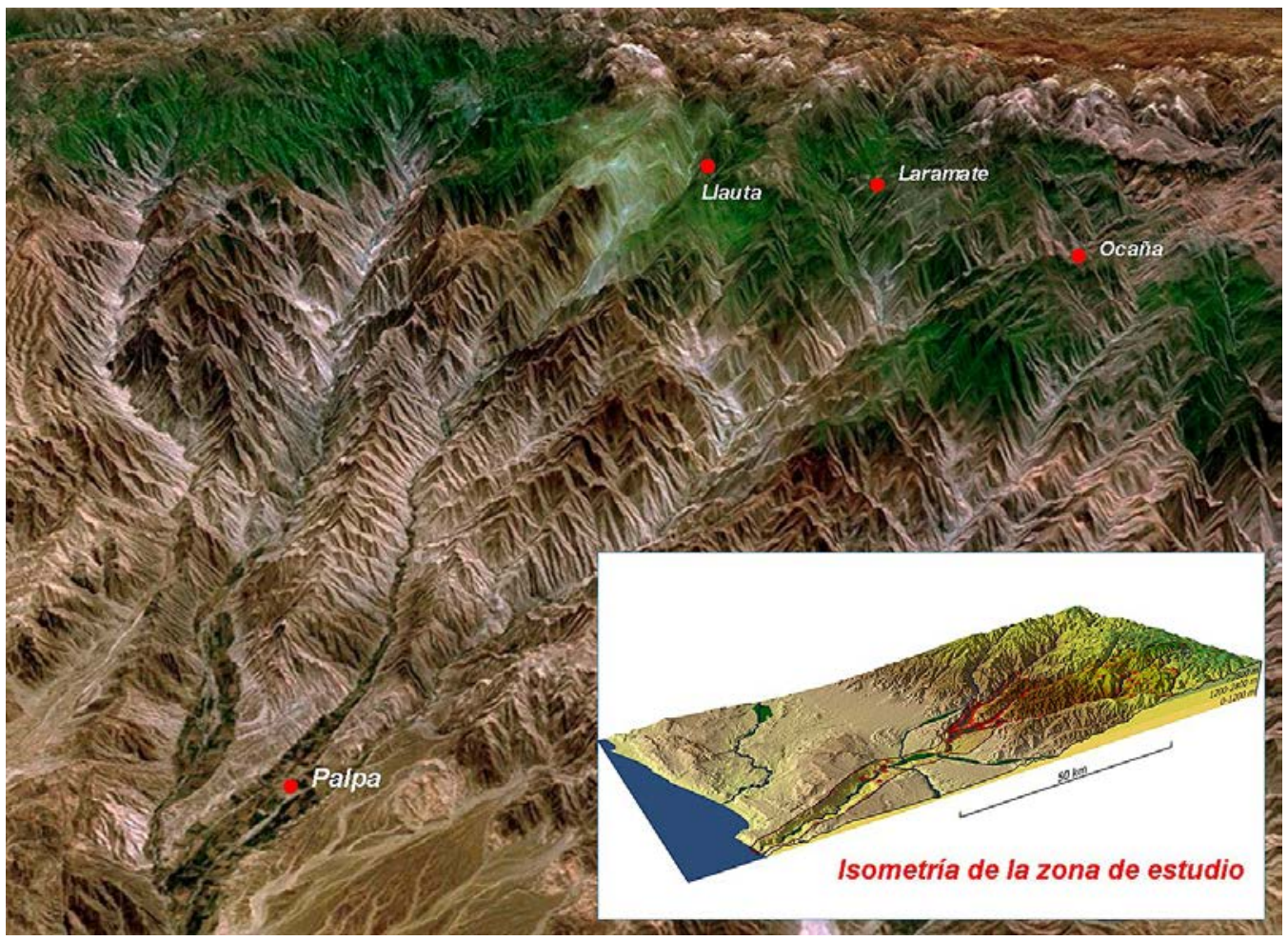

Figura 2. Foto satelital de los valles de Palpa, donde se puede ver la conformación topográfica de la zona de estudio comprendida por el Proyecto "Transecta Andina" (gráfico elaborado por los autores a partir de una foto de Google Earth).

espacios disponibles que se encuentran en las márgenes de los ríos. Por otro lado, por encima de los 3500 metros de altura, el frío es más intenso y las actividades allí solo se limitan al pastoreo en zonas cada vez más reducidas.

\section{Los estudios arqueológicos}

El estudio de los patrones de asentamiento prehispánico en los valles de Palpa, tanto en la costa como en la yunga y la sierra, nos ha permitido registrar repetidamente movimientos poblacionales de este a oeste, es decir, desde la costa árida hacia la sierra y viceversa. Sin embargo, desde el punto de vista arqueológico, la zona de la sierra que se encuentra al este de Palpa era hasta hace poco casi desconocida (Reindel e Isla 2013). En este sentido, después de los estudios en la costa (Reindel 2009; Isla 2009, 2010; ver también Reindel y Wagner 2009), desde 2006, nuestras investigaciones se extendieron hacia la yunga y la sierra hasta los 4350 metros sobre el nivel del mar, y comprendieron las cabeceras de los valles de Palpa, que se encuentran entre los poblados de Llauta, Laramate y Ocaña (Reindel e Isla 2016). ${ }^{3}$

Los trabajos de campo inicialmente comprendieron prospecciones de campo y excavaciones restringidas, y luego también excavaciones en área. De este modo, las prospecciones arqueológicas realizadas en la zona entre los años 2006 y 2009 nos permitieron registrar más de 345 sitios arqueológicos pertenecientes a todos los períodos de tiempo (Fig. 3), ${ }^{4}$ la mayoría de los cuales pertenecen a culturas tradicionalmente consideradas como costeñas, las cuales son Paracas, Nasca e Ica-Chincha, así como también a la cultura Wari de Ayacucho (Reindel 2010, 2012). En este punto, es importante indicar que, a diferencia de la costa, en los sitios de la sierra, es notoria la escasez de materiales 


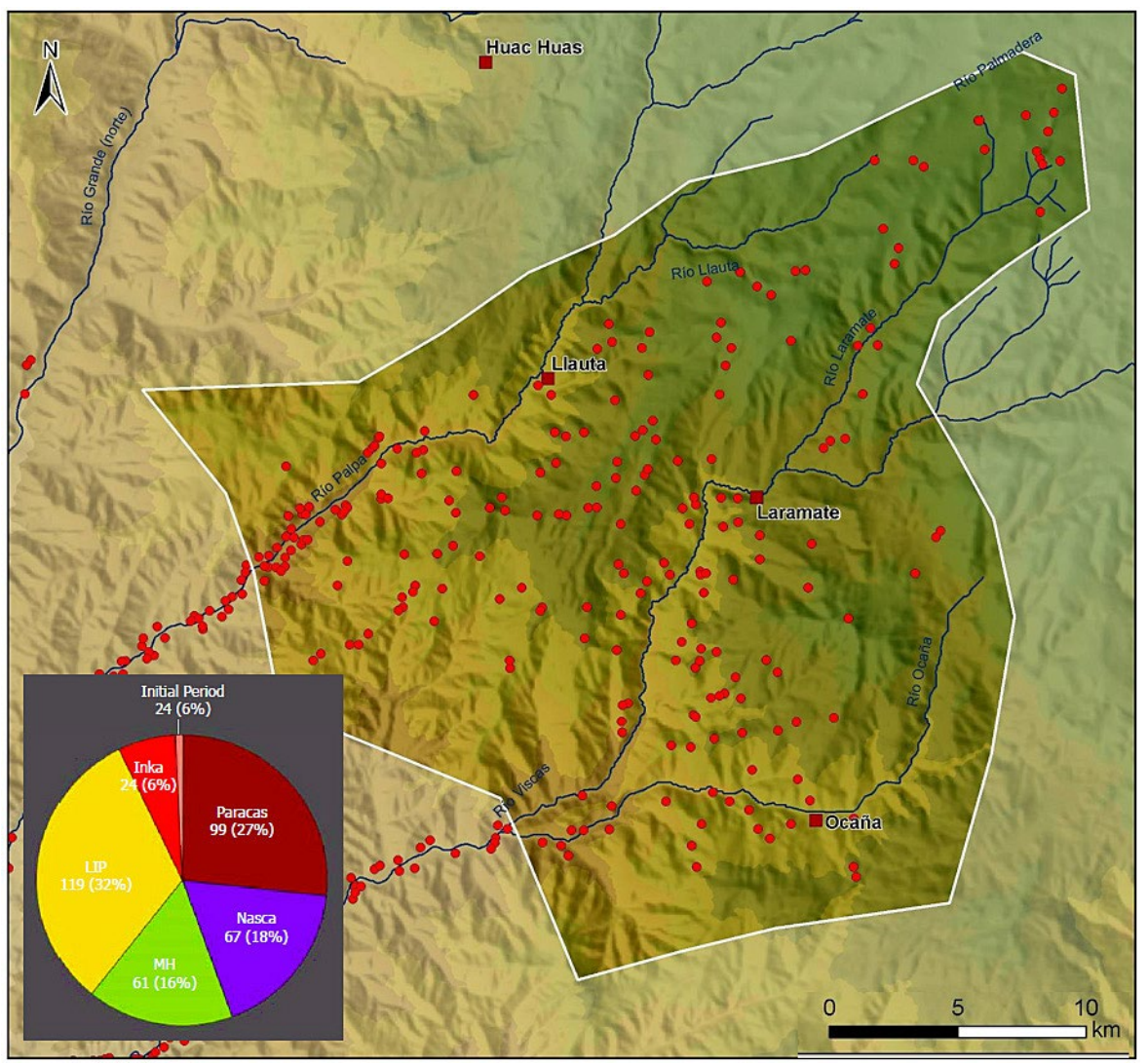

Figura 3. Mapa del área de estudio en la sierra con ubicación de todos los sitios registrados durante los trabajos de rospección entre el 2006 y 2009 (gráfico elaborado por V. Sosna a partir de una foto de Google).

diagnósticos en la superficie, por lo que casi el 20\% del total de sitios registrados no han podido ser asociados a ningún período de tiempo en particular. Pese a esto, según las evidencias registradas, se ha llegado a constatar que la zona empezó a ser ocupada más intensivamente durante el desarrollo de la cultura Paracas, particularmente, en la época Paracas Tardío, con intervalos que muestran una disminución de la ocupación durante el desarrollo de las culturas Nasca y Wari hasta alcanzar su pico más alto durante el Período Intermedio Tardío en relación con la cultura Ica-Chincha. ${ }^{5}$

En este sentido, el análisis de los datos nos ha permitido establecer interesantes paralelos y coincidencias entre el patrón de asentamiento de la costa y de la sierra, en la cual también se incluye a la yunga como zona intermedia.

Por otro lado, los trabajos de prospección en la sierra también nos permitieron identificar otras evidencias que indican que en el pasado hubo condiciones climáticas que permitieron una economía variada y productiva. Así, además de los numerosos sitios arqueológicos registrados, también, se identificaron una gran cantidad de terrazas agrícolas establecidas cerca de los asentamientos, así como en las faldas y laderas de los cerros. ${ }^{6}$ En general, en toda la zona estudiada, se observan grandes complejos de andenes que, vistos en conjunto, conforman un extenso paisaje cultural, lo que contrasta con la baja densidad poblacional que existe en la actualidad: sus habitantes viven de manera dispersa en pequeños caseríos, entre los que destacan los poblados de Llauta, Laramate y Ocańa como los más grandes. ${ }^{7}$

En esta misma perspectiva, se pueden considerar los numerosos corrales antiguos establecidos arriba de los 3000 metros de altura, los cuales son un indicador de que, en tiempos prehispánicos, 


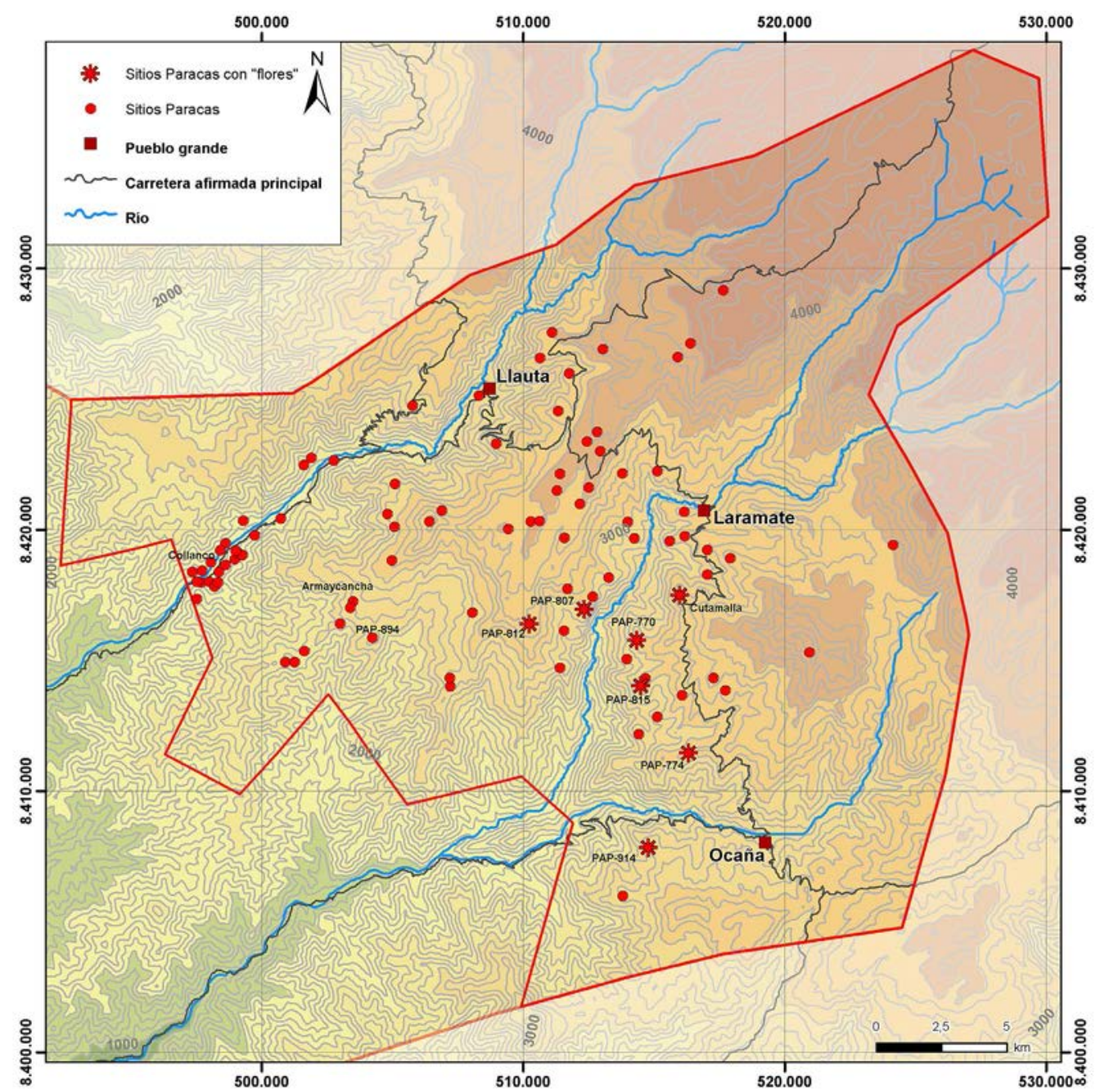

Figura 4. Mapa del área de estudio con la ubicación de todos los sitios de filiación Paracas registrados en la zona de estudio, entre los que destacan los sitios en forma de flor (gráfico: V. Sosna).

la crianza y el manejo de rebaños de camélidos eran de gran importancia para la economía de la zona. No hay que olvidar que, en dicho período, los camélidos eran muy apreciados por su carne y su lana, así como animales de carga. A partir de estas evidencias, se sabe que los habitantes de la sierra tenían una base económica sustentada en la agricultura y la ganadería, cuyos productos luego se intercambiaban con otros de la costa.

En este sentido, también se han identificado rutas de comunicación entre la costa y la sierra que, en la zona de estudio, datan de por lo menos la época Paracas. Dichas rutas facilitaban el intercambio de productos y la movilización de la gente. ${ }^{8}$ Además, estos caminos conectaban importantes yacimientos de obsidiana en la sierra que, luego, eran llevados a la costa (Burger y Glascock 2000; Tripcevich y Contreras 2011). Las fuentes etnohistóricas también refieren que desde la costa se transportaba algodón, ají y otros productos agrícolas hacia la sierra (Guillén 1984). 


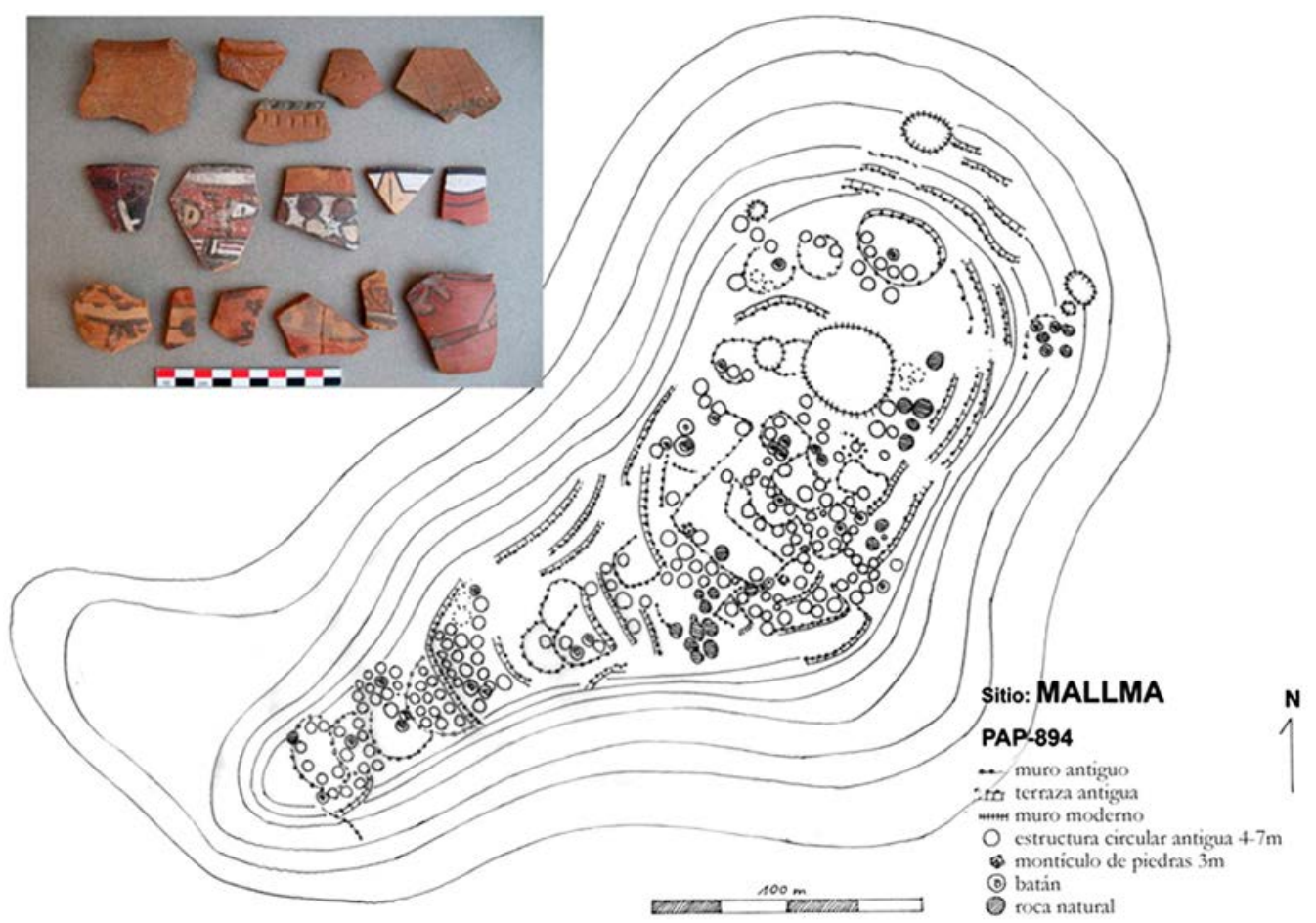

Figura 5. Dibujo esquemático de sitio de Malma (PAP-894), un asentamiento de filiación Paracas ocupado en diferentes periodos de tiempo (dibujo: C. Hohmann; foto: J. Isla).

\section{Los asentamientos Paracas}

Durante los trabajos de prospección en la sierra, se han registrado más de 80 sitios pertenecientes al Horizonte Temprano (Fig. 4), la mayoría de los cuales pertenecen a las épocas Paracas Medio (fases Ocucaje 6-7) y Paracas Tardío (fases Ocucaje 8-9), aunque hay algunos sitios que muestran evidencias de haber sido reutilizados total o parcialmente durante la época Nasca e, incluso, durante el Período Intermedio Tardío. En algunos casos, la ausencia de materiales diagnósticos a veces impide plantear con mayor precisión la posición temporal de los sitios; no obstante, cada vez, se conocen más los rasgos formales y constructivos de los sitios, y, por tanto, se pueden distinguir mejor los rasgos comunes para cada período de tiempo.

Los sitios registrados ocupan diversos espacios de la zona estudiada, en especial, las márgenes altas a la derecha e izquierda del río Laramate, y cerca del camino de larga distancia. Se trata principalmente de asentamientos pequeńos de tipo doméstico, entre los que destacan algunos sitios más grandes, como Mallma (PAP-894), los cuales ocupan las cimas de lomas o colinas, y se ubican cerca de los grandes complejos de terrazas agrícolas y de las fuentes de agua.

El sitio de Mallma, el cual se encuentra al inicio de la zona más húmeda, en la cima de una lomada cerca del caserío de Armaycancha, es uno de los más característicos de este tiempo, y destaca porque presenta un gran número de estructuras de planta circular u ovalada, hechas con muros de piedra de doble cara, que se distribuyen en grandes patios y amplias terrazas (Fig. 5). ${ }^{9}$ Cabe indicar que las estructuras ubicadas en la parte alta del sitio son más grandes, mejor elaboradas y con mayor espacio entre sí, mientras que las que se localizan en la parte más baja —al lado sur y sudoeste— son más sencillas y están más aglutinadas. Es posible que estas diferencias estructurales estén reflejando una cierta jerarquía social de la comunidad. 
Además de los sitios antes descritos, se registró un grupo de sitios correspondientes a corrales de pastores que aprovechaban las zonas con pastos de la zona Puna y Suni, arriba de los 3500 metros sobre el nivel del mar, las cuales son aptas para la crianza de camélidos. Cerca de estos corrales, se encuentran los cimientos de pequeñas estructuras de planta circular u ovalada que seguramente eran ocupadas por los pastores, tal como ocurre hoy en día en otros lugares. Los sitios pastoriles se encuentran dispersos en toda la zona alta alrededor de Llauta, Laramate y Ocańa, así como en la meseta entre los sistemas fluviales y en el altiplano.

\section{Sitios Paracas en forma de flor}

Además de los sitios antes descritos, durante los trabajos de prospección, también se identificaron varios sitios que tenían rasgos constructivos particulares: recintos en forma de «D» dispuestos en grupos de dos, tres o más alrededor de un espacio abierto o — en el mejor de los casos— alrededor de un patio, formando construcciones que vistos de planta se parecen a una flor. La mayoría de estos sitios (PAP 667, 770, 807, 812 y 815) se localizan en ambos lados del río Laramate, y ocupan lomas y colinas prominentes que se interconectan visualmente. Solo dos de ellos (PAP-774 y 914) se encuentran más al sur, en ambas márgenes del río Ocańa (Fig. 4), y ocupan una posición prominente desde donde también están conectados visualmente con los otros.

En la mayoría de casos, estos sitios cuentan con dos sectores bien definidos. Uno es de aparente uso doméstico y presenta un recinto de planta ovalada, circular o en forma de «D», que está dispuesta de manera de concentrada y alternada con espacios más grandes a modo de patios. El otro es de aparente uso público, correspondiente a las estructuras con patios hundidos y recintos en forma de «D» que se describen arriba; dichas estructuras ocupan espacios más grandes, son más sólidas y de mejor acabado. Por los rasgos formales y constructivos, este tipo de construcciones pueden ser consideradas como monumentales para la zona. El sitio más grande y representativo de este tipo es Cutamalla (PAP-767), el cual se describe más adelante y en el cual además se han realizado excavaciones. A continuación, se presenta una muy breve descripción de las estructuras arquitectónicas en forma de flor presentes en la zona de estudio.

\subsection{Sitio PAP-767 (Cutamalla)}

El sitio presenta dos sectores, uno de carácter habitacional (sector A) localizado en la parte más alta, en el lado sur del sitio, y otro de carácter público o ceremonial (sector B), ubicado en la parte más baja, en el lado norte del sitio (Fig. 6). Entre ambos sectores y de manera especial en todo el lado oeste del sitio, se encuentra un numeroso conjunto de terrazas agrícolas que siguen la topografía inclinada del terreno.

Las construcciones en el sector A comprenden los cimientos y muros de una serie de recintos de piedra de planta ovalada, circular y en forma de «D», las cuales se disponen en forma aislada o en grupos separados por patios y grandes espacios abiertos siguiendo la topografía del terreno. La mayoría de las estructuras arquitectónicas en este sector han sido afectadas por la construcción de corrales actuales. Por otro lado, en el sector B, se encuentra un grupo de 10 estructuras arquitectónicas bastante grandes, que, según sus rasgos constructivos, presentan una planta en forma de flor (Fig. 7). ${ }^{10} \mathrm{La}$ estructura arquitectónica más completa está conformada por un patio hundido de contorno circular, alrededor del cual se encuentran una serie de recintos en forma de "D» que tienen una pequeña terraza delante del muro recto, orientada hacia el patio hundido.

\subsection{Sitio PAP-770 (Qechqalla)}

En el sector A del sitio, ocupando la cima de una colina, se encuentran tres estructuras en forma de flor, cada una con una plaza semihundida de forma casi circular, rodeada por 11 y 12 recintos en forma de «D». En comparación con las estructuras en forma de flor de Cutamalla, las plazas hun- 


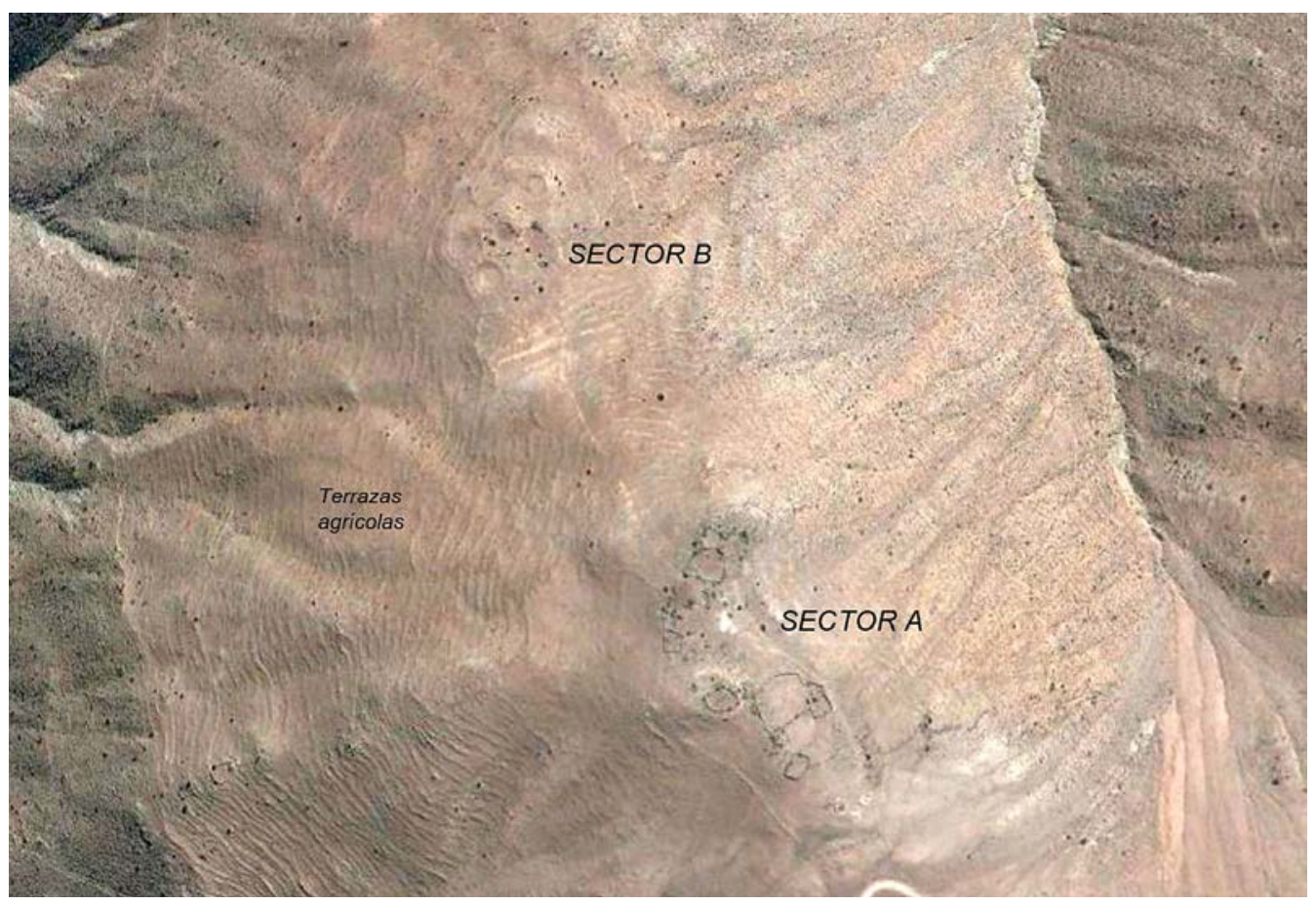

Figura 6. Foto satelital del sitio de Cutamalla (PAP-767), en donde se pueden observar los dos sectores que conforman el sitio y las numerosas terrazas de cultivo (fuente: Google Earth 2013).

didas son menos regulares y presentan algunas estructuras o subdivisiones internas. En algunas de estas estructuras, se registraron batanes. La cerámica registrada en este sitio data de la fase Ocucaje 8 , con lo cual se constituye como el sitio de este tipo más temprano registrado hasta el momento.

\subsection{Sitio PAP-774 (Musuq Cancha)}

En este sitio, se encuentran una serie de terrazas bien adaptadas a la pendiente de la colina y cinco estructuras en forma de flor localizadas en ellas, cada una con un número variable de cuatro a 15 recintos en forma de «D» dispuestos alrededor de un patio central. El modelo arquitectónico es similar al de Cutamalla, pero están en mal estado de conservación. Los recintos en forma de «D» están elaborados con muros que incluyen piedras seleccionadas unidas con mortero de barro, los cuales miden 4,5 por 6 metros de lado y presentan buenos paramentos. En todas las plazas hundidas, se registraron batanes, pero muy pocos fragmentos de cerámica.

\subsection{Sitio PAP-807}

En este sitio, se distinguen al menos seis estructuras en forma de flor con patios hundidos, dos de forma circular y cuatro en forma de media luna, todas con recintos en forma de «D" dispuestas alrededor a modo de pétalos. La última estructura se localiza en la parte más baja del sitio y tiene solo tres recintos en forma de «D».

\subsection{Sitio PAP-812 (Chaqui Cocha)}

El sitio ha sido dividido en cuatro sectores: A, B, C y D. En el sector A, se encuentran cinco estructuras hundidas de forma circular o en forma de media luna, con un diámetro variable de 10 a 15 metros, con tres recintos en forma de «D» dispuestas alrededor de la estructura circular. 


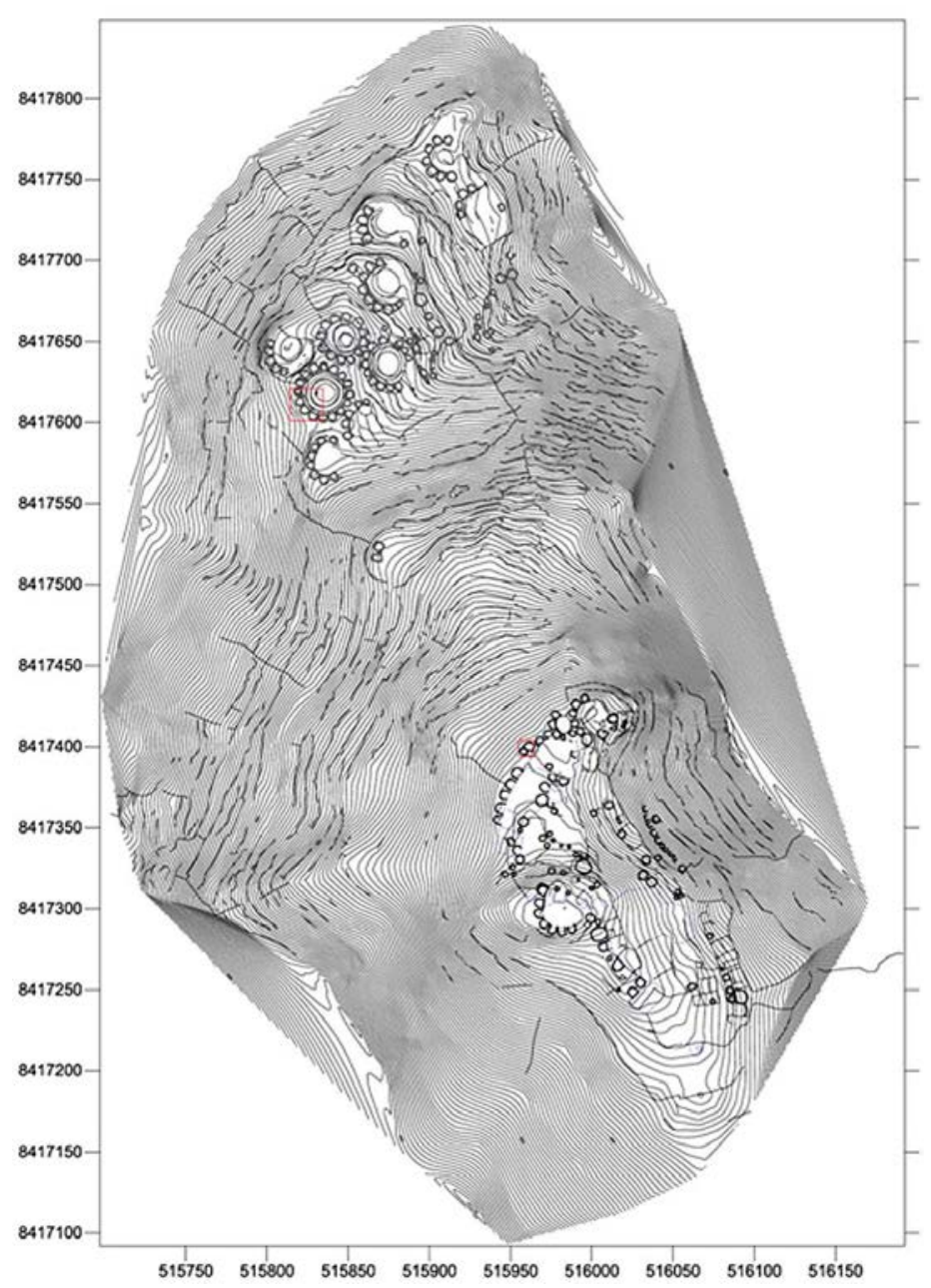

Figura 7. Mapa topográfico y arquitectónico del sitio de Curamalla, en el que se puede observar con mayor claridad las estructuras arquitectónicas que forman parte del asentamiento (dibujo: M. Reindel).

En la parte baja del sector B, se encuentra otros cuatro patios semicirculares hundidos con tres recintos en forma de «D» alrededor. Finalmente, en el sector $\mathrm{C}$, que ocupa una loma alargada, se encuentra un patio hundido de contorno circular con tres recintos en forma de "D» a su alrededor.

\subsection{PAP-815 (Huayuncalla)}

En la parte superior del sitio, se puede distinguir un patio circular hundido con una estructura central, rodeada de pequeños recintos en forma de «D» que miden de 4 por 5 metros de lado distribuidos de manera regular.

\subsection{PAP-914 (Morocolla)}

En este sitio, destacan una serie de estructuras de vivienda que tienden a tener forma de flor, es decir, recintos en «D» dispuestos alrededor de espacios abiertos o de media luna; sin embargo, en la parte más alta del sitio, se encuentran tres estructuras mejor definidas en forma de flor que son más parecidas a las de Cutamalla, aunque en este caso no presentan el patio interior. En este caso, 


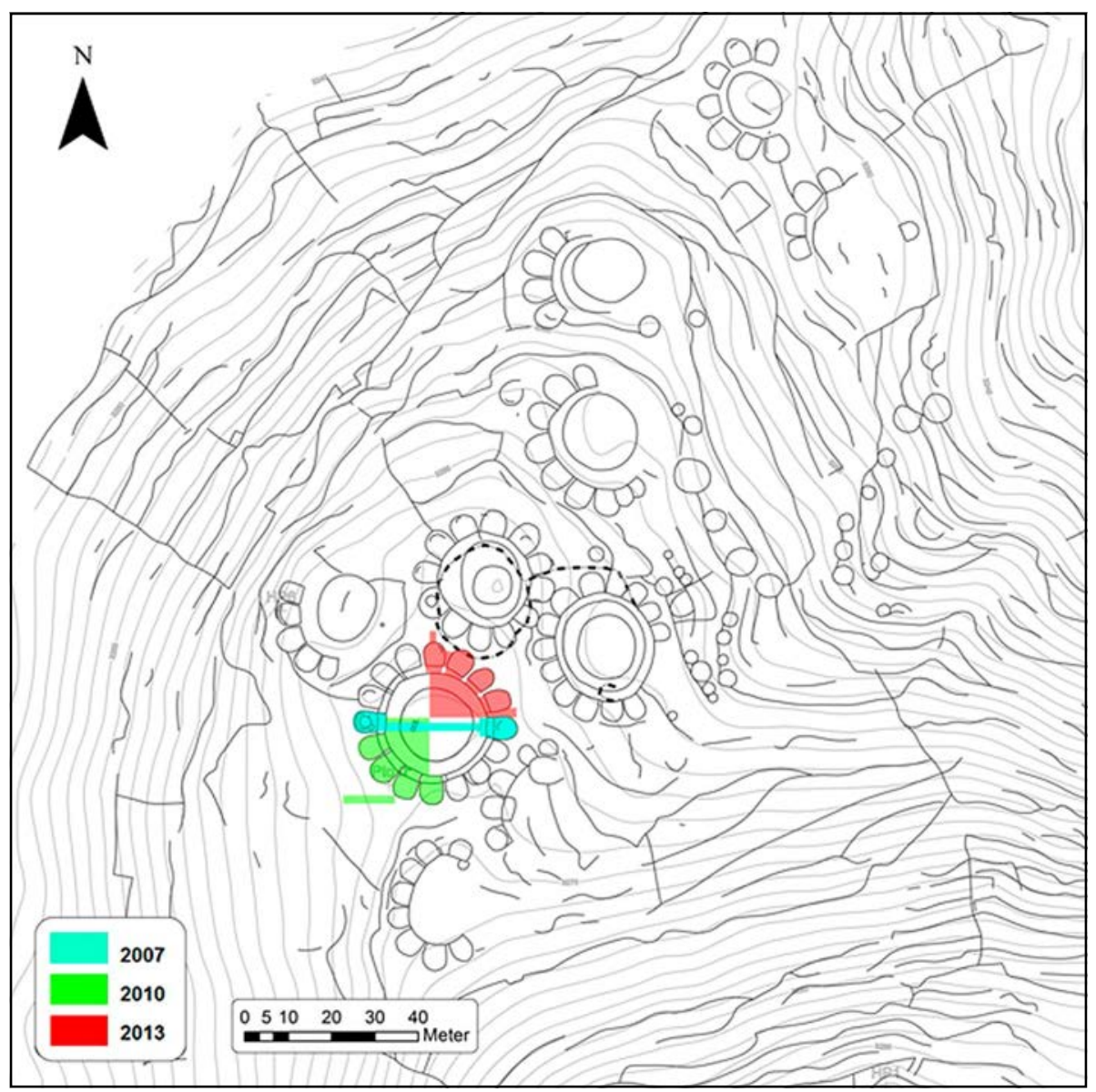

Figura 8. Plano en detalle del grupo de estructuras arquitectónicas en forma de flor que se encuentran en el sector $B$ de Cutamalla. Nótese la estructura más completa en la que se realizaron las excavaciones (dibujo: M. Reindel).

los recintos en «D» están hechos con una hilera de grandes piedras de campo, las cuales en algunos casos tienen hasta dos o tres hiladas.

\section{Las excavaciones en Cutamalla}

Las excavaciones en Cutamalla — en el sector B — comprendieron a una de las estructuras arquitectónicas en forma de flor, la más completa registrada en el sitio y tal vez una de las más representativas de todos los sitios de este tipo en la sierra de Lucanas. Las excavaciones se realizaron, primero, mediante una trinchera bastante grande que cortaba toda la estructura casi de este a oeste, y, luego, mediante excavaciones en área que comprendieron los cuadrantes noreste y sudoeste, respectivamente (Fig. 8). De este modo, se llegó a exponer un área de más de 800 metros cuadrados que corresponde a un poco más de la mitad de toda la estructura arquitectónica y parte de su contorno exterior, la cual mide unos 40 metros de diámetro en total.

Las excavaciones comprendieron parte del patio central hundido, parte del espacio inclinado libre de construcciones y 10 de los 16 recintos en forma de «D» que definen el contorno de la estructura en forma de flor (Figs. 9 y 10). Seguidamente, se presenta un resumen de los resultados obtenidos en las excavaciones: primero, el patio hundido (UA-7); luego, el espacio inclinado (UA-6); $y$, finalmente, los recintos en forma de «D»(UA-1 a 5 y 8 a 12). 


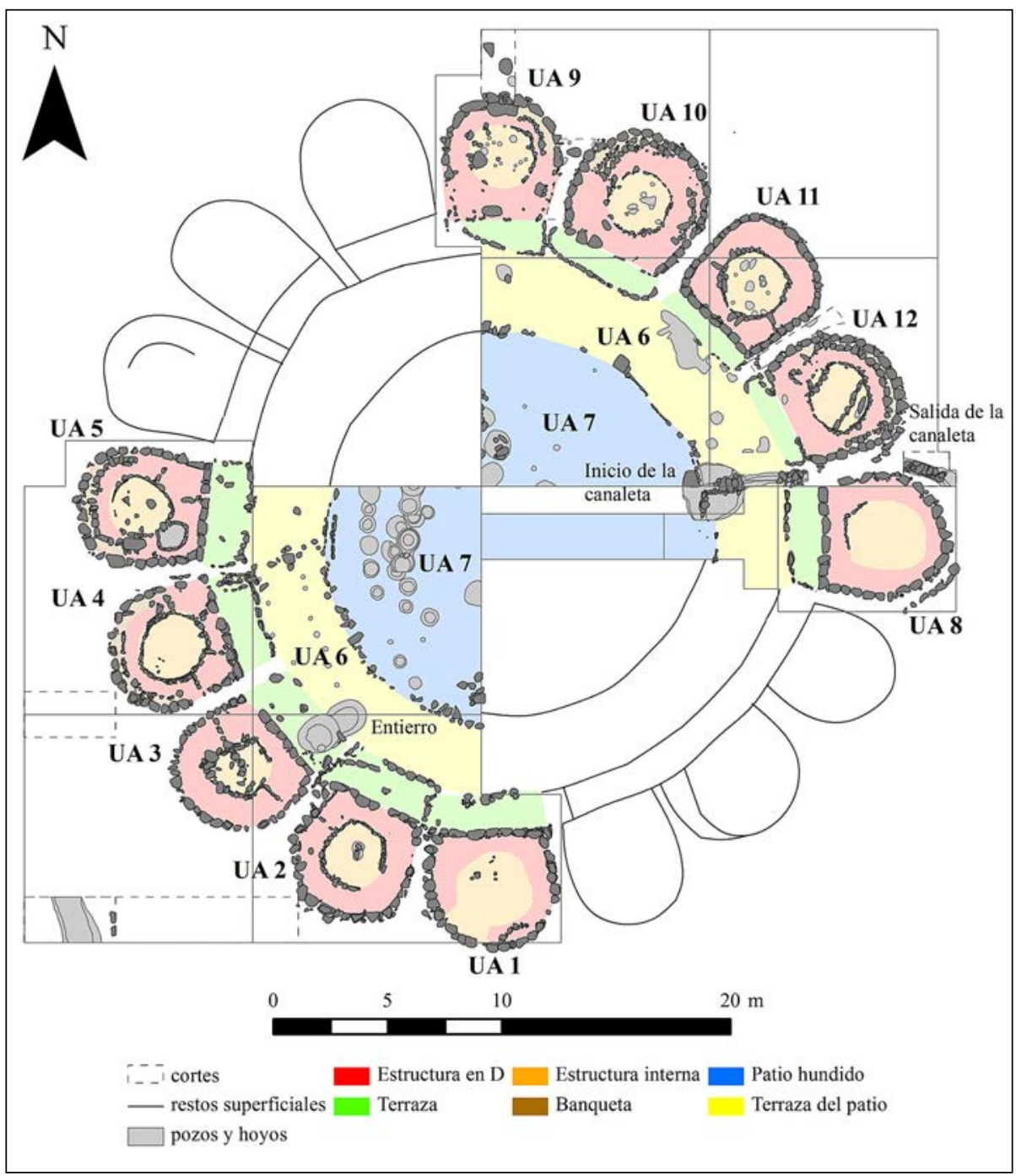

Figura 9. Plano en detalle de la estructura arquitectónica más completa que se encuentra en el sector B de Cutamalla, en donde se muestra las secciones excavadas en las tres temporadas de campo (gráfico elaborado por los autores).

\subsection{El patio hundido (UA-7)}

El patio hundido corresponde al espacio central de la estructura arquitectónica en forma de flor, el cual fue construido en un lugar donde el terreno era relativamente plano, pero con un ligero declive hacia el este y noreste, en donde fue rellenado artificialmente hasta alcanzar un nivel casi horizontal. Sobre este nivel, luego, se construyeron los recintos en forma de «D». De este modo, el patio hundido en gran parte fue construido excavando el terreno natural, conformado por tierra arcillosa compactada hasta llegar a una capa más dura de roca granítica, sobre la cual se formó una superficie casi horizontal.

El patio hundido es de forma casi circular, mide 16,5 metros de diámetro, y estaba delimitado por un muro hecho a partir de una hilera de piedras grandes y alargadas que fueron colocadas en forma vertical, con sus lados más planos dispuestos hacia el interior del patio, de tal modo que presentaba un acabado regular y más vistoso (Fig. 11). Dicho muro se dispuso a manera de enchape sobre la pared formada por el terreno, tenía 15 a 20 centímetros de ancho y 60 centímetros de 


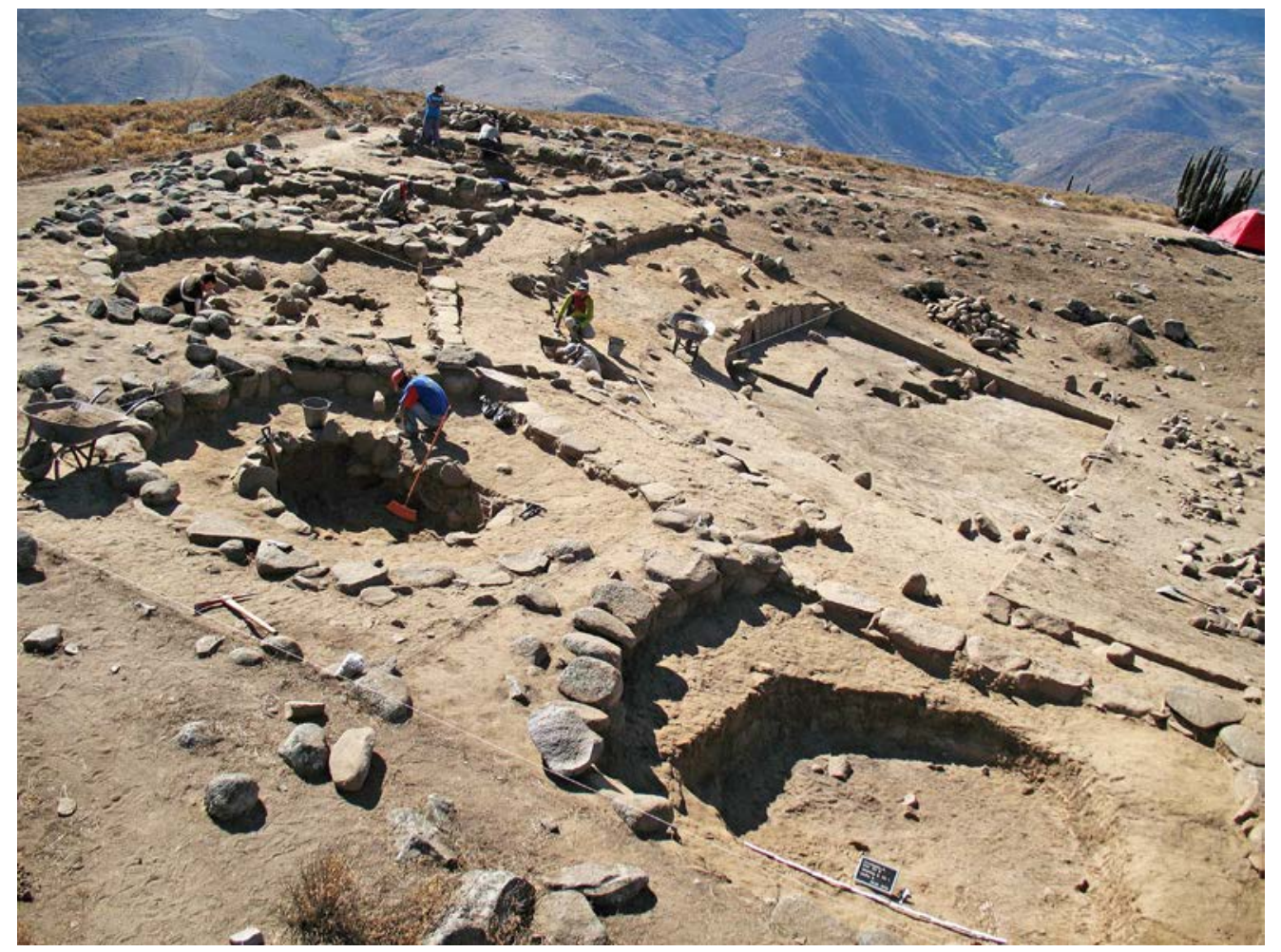

Figura 10. Vista panorámica de las excavaciones en el cuadrante suroeste de la estructura arquitectónica en forma de flor. Nótese los recintos en "D» dispuestos alrededor del patio hundido (foto: J. Isla).

altura promedio, y era completado en partes con piedras pequeñas y medianas para mantener un mismo nivel. Las piedras fueron asentadas directamente en el terreno; en algunos casos, se notan pequeñas piedras dispuestas como cuñas. En su interior, el patio presentaba un piso casi plano que se encontraba casi al nivel de la base del muro.

Dicho piso, en realidad, corresponde a un apisonado regular y compacto que se formó sobre una delgada capa de tierra dispuesta sobre la capa natural. En relación con el apisonado, se encontraron fragmentos de cerámica de la fase Ocucaje 9, varias puntas y lascas de obsidiana, así como también alisadores y piruros de cerámica.

Debajo del apisonado, al nivel de la superficie de la capa natural, se registraron una serie de matrices circulares de uno a 1,5 metros de diámetro, que resultaron siendo silos o pozos profundos de forma cónica excavados en la capa natural. Estos se encuentran mayormente en su lado oeste, y forman dos filas más o menos alineadas que se disponen de manera paralela al muro del patio hundido (Fig. 11). Todos los pozos estaban rellenados con tierra arenosa fina y suelta de color marrón, la cual en la mayoría de casos tenía residuos de carbones y restos de tierra quemada. Asimismo, se nota que las paredes interiores de algunos de ellos habían sido parcialmente revestidas con una y dos delgadas capas de barro.

Finalmente, en el lado este del patio hundido, se descubrió una canaleta de drenaje que, según las evidencias estratigráficas, se construyó algún tiempo después de haber terminado con la construcción de los recintos en «D». Esto se deduce porque el curso del canal pasa justo entre dos recintos en «D» (UA-8 y 12), cortando las capas de relleno sobre las cuales se asentaban dichos recintos.

La canaleta mide en promedio 15 a 20 centímetros de ancho, 30 centímetros de profundidad y 10,8 metros de largo, y presenta un declive moderado que va de oeste a este. Como se dijo, la canaleta se realizó excavando el terreno natural y gran parte de su trayecto estaba cubierto por lajas 


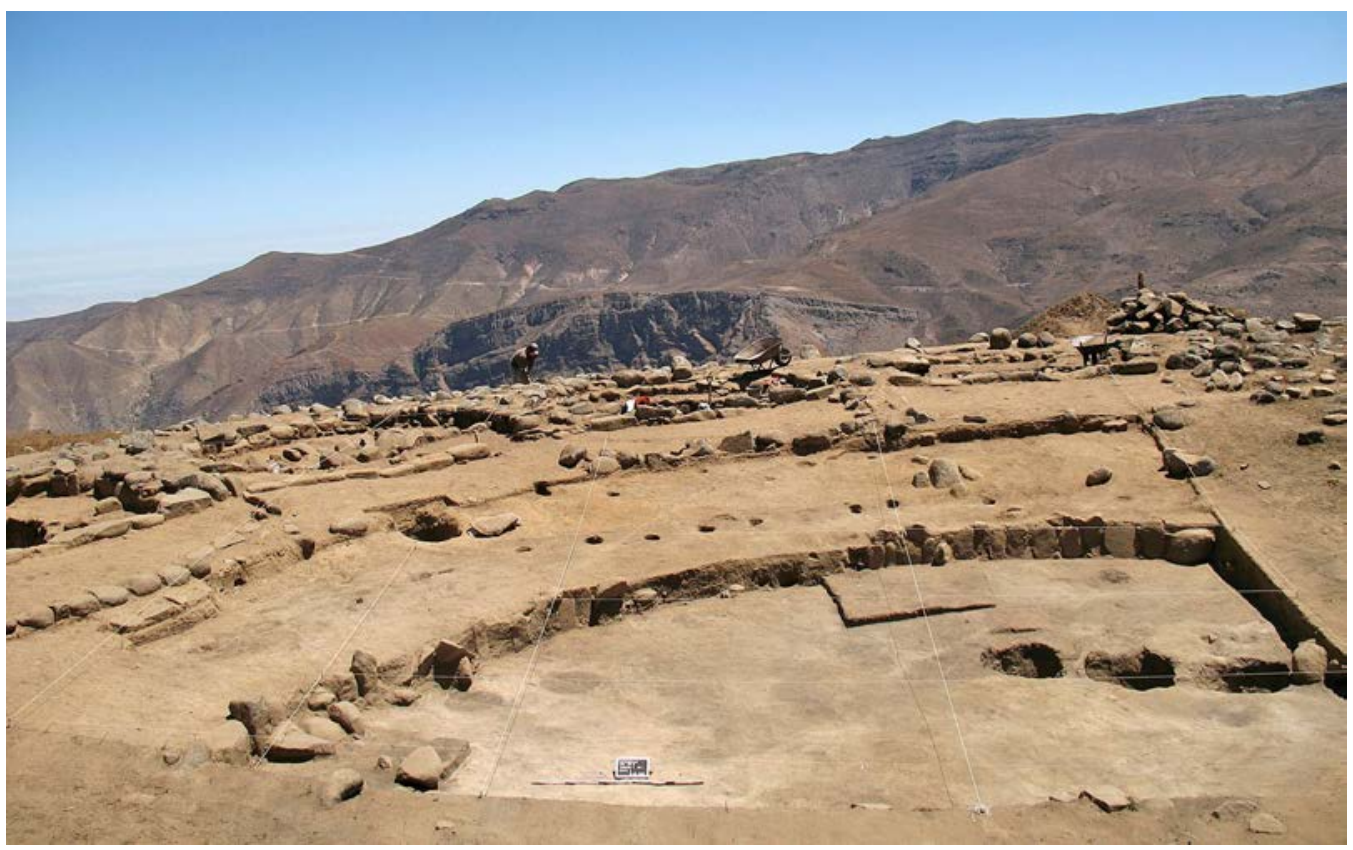

Figura 11. Vista general de las excavaciones con primer plano del patio hundido delimitado con un muro de lajas, en cuyo interior se observan tres silos alineados. En el espacio intermedio, también, se observan algunos hoyos de postes (foto: J. Isla).

que estaban unidas con un mortero de barro arcilloso que tenía una consistencia dura y compacta (Fig. 12). Al parecer, esta canaleta de drenaje se construyó por la necesidad de evacuar el agua acumulada al interior del patio hundido durante épocas de fuertes lluvias, cuando no fue posible hacerlo manualmente con el uso de ollas o cántaros. En este sentido, se debe recordar que el fondo del patio está compuesto por estratos duros de roca granítica y sedimentos arcillosos impermeables.

\subsection{El espacio inclinado (UA-6)}

Más que una estructura arquitectónica, se trata de un espacio libre de construcciones que se encuentra alrededor del patio hundido, delimitado por el muro de este y los alineamientos de piedras que delimitan las pequeńas terrazas que se encuentran delante de los recintos en forma de «D" (Fig. 9). Dicho espacio tiene tres metros de ancho promedio y una inclinación de unos $30^{\circ}$ a $45^{\circ}$ en dirección al patio hundido.

Las excavaciones en el cuadrante sudoeste permitieron exponer la capa natural a pocos centímetros de la superficie, mientras que, en el cuadrante noreste - y también el lado este-, la situación era muy distinta debido al declive que presentaba el terreno en esa dirección. En este sentido, en esa zona, en lugar de rebajar el terreno natural, se agregaron varias capas de relleno para aumentar su altura y obtener el mismo nivel que el resto del conjunto, de tal modo que todos los recintos en «D» se encuentran más o menos al mismo nivel.

En general, las excavaciones en esta parte de la estructura arquitectónica permitieron registrar algunos pocos rasgos que destacar, entre ellos, un contexto funerario (CF-1) encontrado en el cuadrante sudoeste, justo debajo del alineamiento que delimita la terraza de la UA-3. La matriz corresponde a un pozo en forma de bota, en cuyo interior se encontraban los restos humanos muy mal conservados y se halló solo una punta de obsidiana como ofrenda. Según la posición estratigráfica, se puede afirmar con bastante certeza que se trata de un contexto de filiación paracas establecido en algún momento de ocupación del sitio. 


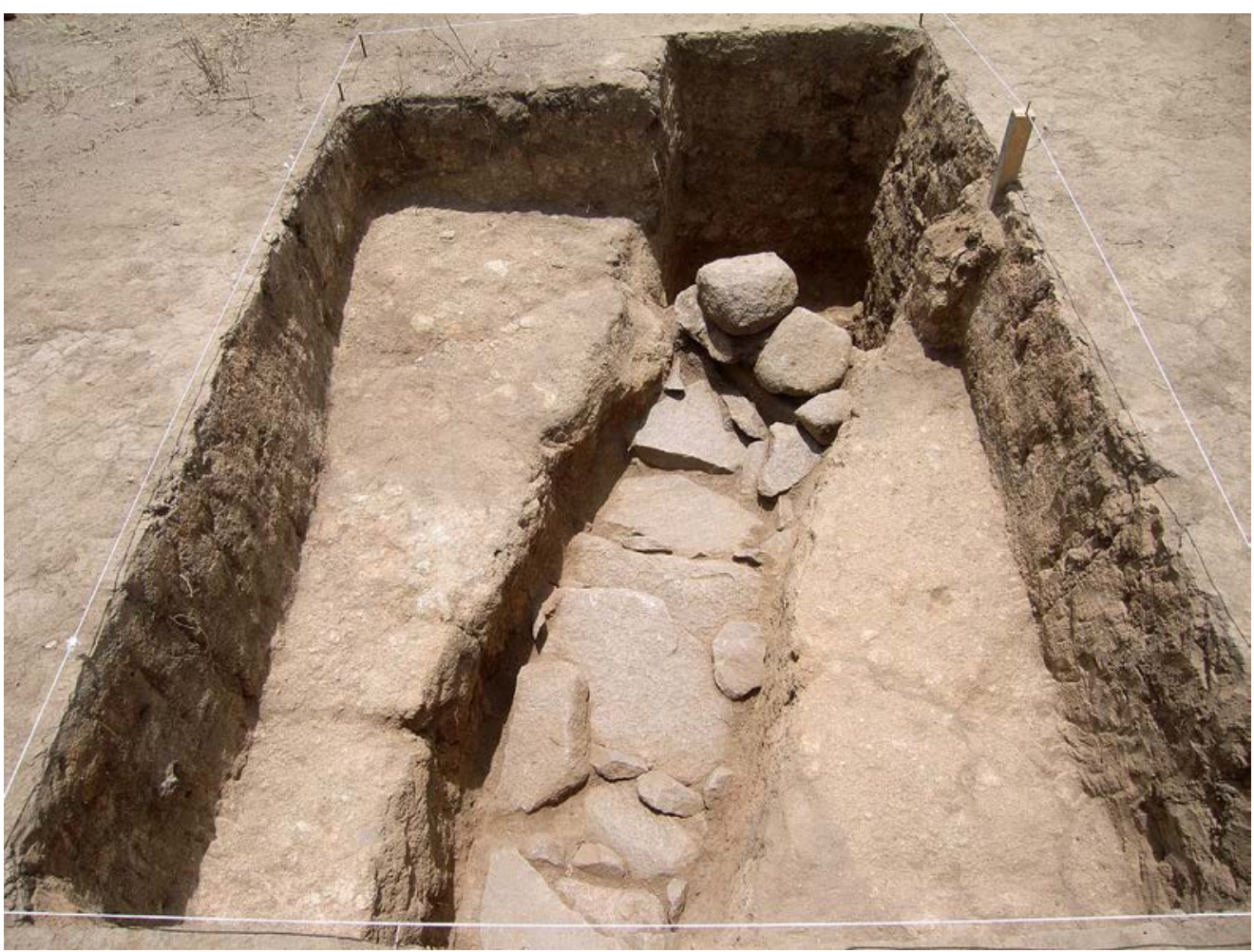

Figura 12. Primer plano de la canaleta descubierta entre las UA-8 y 12, a través de la cual se evacuaba el agua que se empozaba en el patio hundido. Nótese las lajas estaban unidas con mortero de barro (foto: J. Isla).

Por otro lado, siempre en el cuadrante sudoeste, en relación con la superficie del terreno natural, se llegó a notar varios lentes alargados y círculos de color gris. Al limpiarlos, se descubrió una delgada zanja y hoyos que fueron excavados en la capa natural (Fig. 11). En total, se identificaron 11 hoyos que tenían entre 12 a 20 centímetros de diámetro, ocho de los cuales estaban más o menos alineados en forma casi paralela al muro del patio hundido, en la parte media de este espacio inclinado. Al parecer, se trata de hoyos de postes que sirvieron para sostener un techo liviano, el cual se encontraba solo en algunas partes de dicho espacio.

\subsection{Los recintos en forma de «D»}

Alrededor del patio hundido y del espacio inclinado, se encuentran 16 recintos en forma de «D», todos los cuales presentan delante de sí un pequeño espacio de forma rectangular delimitado con un alineamiento de piedras medianas, que se interpreta como terrazas orientadas hacia el patio hundido. Los recintos en «D" se encuentran a más o menos 1,40 metros sobre el nivel del patio hundido y forman una especie de anillo alrededor del mismo (Fig. 9). Las excavaciones comprendieron a 10 recintos en los dos cuadrantes opuestos, los cuales, aunque diferentes en algunos detalles constructivos, así como en la composición de algunos rasgos interiores, son muy similares en forma, tamaño y orientación con respecto al patio hundido. Debido a ello, aquí se realiza una descripción conjunta de los mismos, haciendo énfasis en sus particularidades y otros rasgos relevantes.

Cada uno de los recintos mide en promedio entre 4,5 a cinco metros de ancho y entre cinco y 5,5 metros de largo (Fig. 13). Vistos de planta los recintos tienen una forma de "D» que está compuesta por dos muros: uno recto que se mira hacia el patio hundido y otro de contorno curvo que define la forma de «D». Estos muros están hechos con una hilera de piedras de campo bastante 


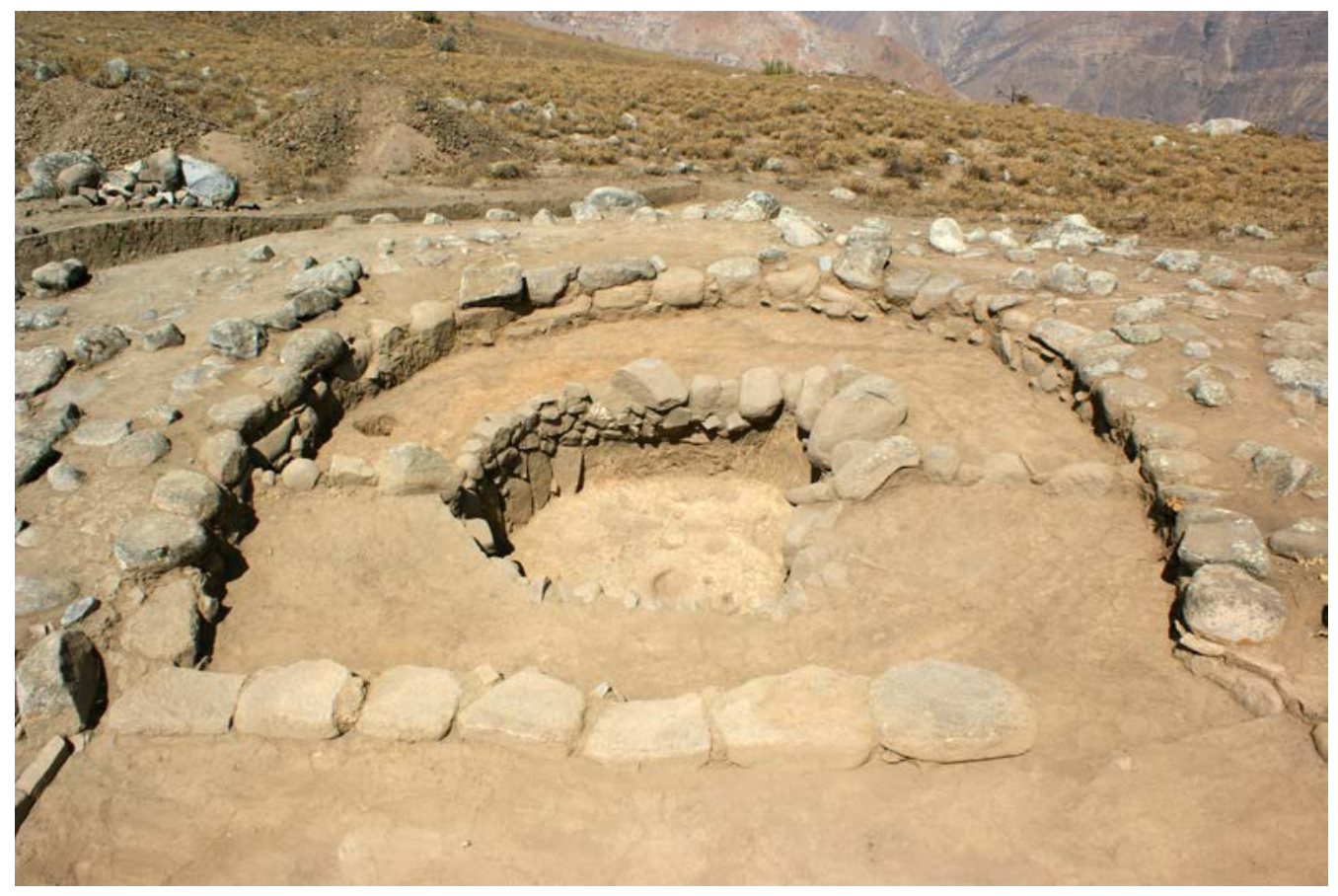

Figura 13. Primer plano de uno de los recintos en forma de "D», en donde se puede ver su conformación arquitectónica y los detalles constructivos de los muros. En la parte central, destaca el recinto hundido (foto: J. Isla).

grandes, que, en la mayoría, de los casos conservan hasta dos hiladas. No tienen mortero, pero, en las bases, se han colocado pequeñas piedras dispuestas como cuñas para estabilizar las piedras grandes. Dependiendo del tamaño de las piedras, los muros miden entre 30 y 50 centímetros de ancho y de altura; cabe anotar que esta parece haber sido su altura original. Siendo esto así, se puede suponer que las paredes y los techos de estos recintos fueron complementados de algún modo con materiales livianos perecibles, de los cuales, sin embargo, no se encontró ninguna evidencia. El acceso a los recintos en "D» no es claro, pero es probable que se haya utilizado el muro recto, que casi en todos los casos solo tenía una hilada de piedras.

Como ya se mencionó previamente, delante de los recintos en «D» se encuentra un espacio rectangular de 1,5 metros de ancho por 4,5 metros de largo, delimitado por un delgado alineamiento de piedras, que parece que funcionó como una pequeña terraza que está orientado hacia el patio hundido. En dichas terrazas, se identificó un solo nivel de uso, lo que indica que las terrazas tenían poco uso o que estaban limitadas a ciertas actividades.

Volviendo a los recintos en "D», se debe indicar que, desde la superficie, se notaba al interior de algunos de ellos una especie de banqueta delimitada por una hilera de piedras, la cual estaba dispuesta en paralelo a la sección más curvada de los muros. En un solo caso (UA-5), la banqueta se encontraba asociada con un gran batán y gran cantidad de fragmentos de cerámica. Según las evidencias registradas, estas banquetas se establecieron en relación con un último momento de ocupación del sitio, durante la época de transición de Paracas a Nasca, cuando los recintos en «D» fueron rellenados y algunos se reutilizaron como espacios de uso doméstico.

Un rasgo importante y común a todos los recintos en «D» es la presencia de un pequeño recinto hundido de contorno circular, el cual se hizo excavando la capa natural en la parte central del recinto, un poco más abajo del nivel de la base de los muros (Fig. 13). Estos recintos miden entre 2,5 y 2,7 metros de diámetro, y tienen entre 60 y 80 centímetros de profundidad. La ubicación central y equidistante con respecto al recinto en «D» indica que estos recintos hundidos formaban parte del mismo diseño constructivo, y que, por lo tanto, se construyeron simultáneamente. Las paredes 
de estos recintos tienen diferentes acabados. En principio, están formadas solo por la superficie vertical de la capa natural, pero, en la mayoría de los casos, presentan una hilera de piedras alargadas, tipo lajas, las cuales fueron colocadas en posición vertical con sus lados planos dispuestos hacia el paramento exterior con la finalidad de obtener un acabado regular. No obstante, en varios casos, se notan combinaciones que incluyen parte de un muro de piedras con la pared natural.

En el fondo de algunos recintos hundidos, se registraron partes de apisonados en los que había residuos de tierra quemada y carbones, así como también hoyos y pozos pequeños rellenados con tierra oscura y carbones. En la UA-9, también, se encontró un batán bastante grande en el fondo. Solo en la UA-1 se encontraron dos niveles de uso superpuestos, sobre los cuales había muchos restos de carbones, tierra quemada y fragmentos de cerámica, lo que sugiere que, en ese recinto, se realizaron labores domésticas. No obstante, en algunos recintos, no había ningún apisonado o huellas de uso, sino solamente la superficie trabajada del terreno natural, lo que sugiere que estos espacios pudieron haber sido utilizados como depósitos.

En estos recintos, se encontraron fragmentos de cerámica, en la mayoría de los casos, de tipo utilitario. Entre ellos, había algunos fragmentos incisos de la fase Ocucaje 9, así como artefactos de cerámica (piruros, pulidores y alisadores) y artefactos líticos (cuchillos y puntas de obsidiana). En algunos casos, estos objetos estaban depositados al interior de los hoyos a modo de ofrenda. De cualquier forma, lo que resulta evidente es que el fondo de los recintos hundidos constituyó un primer nivel de uso de los recintos en «D», el cual funcionaba en relación con otro apisonado que se encontraba más arriba, al nivel de la superficie del recinto hundido.

El acceso a la mayoría de los recintos hundidos se realizaba a través de un podio o escalón conformado por una piedra plana y alargada, la cual estaba colocada en posición vertical apoyada en la pared del recinto. Dicha piedra estaba asentada en la capa natural, y consolidada con barro y pequeñas piedras, y estaba apoyada en la pared que se encuentra en dirección del muro recto del recinto en «D», con lo cual se refuerza la idea de que el acceso a dicho recinto se hacía por ese lado. Solo en dos casos (UA-1 y UA-5) se han registrado pequeños espacios en forma de herradura excavados en la capa natural, los lados que servían como una especie de escalón para ingresar o salir del recinto hundido.

Alrededor de los recintos hundidos, se identificó un apisonado duro y compactado, en partes trabajado en la capa natural, que conforma un espacio plano y regular entre el recinto hundido y el recinto en «D». En la mayoría de los casos, dicho espacio presenta subdivisiones internas hechas con delgados alineamientos de piedras, de tal modo que el apisonado alrededor del recinto hundido está dividido en dos, cuatro o más partes simétricas bastante particulares (Fig. 9). Al no haber diferencias importantes en los materiales recuperados en estos espacios, al parecer, se trata más de un detalle decorativo que funcional, el cual se relaciona con el primer momento de ocupación de los recintos en «D».

La estratigrafía relacionada con estos recintos comprende una secuencia de cuatro a cinco capas, en las que se ha identificado claramente dos momentos de ocupación. El primer momento se relaciona con el uso de los recintos hundidos y el apisonado con subdivisiones internas que estaba en la parte superior, entre el recinto hundido y el recinto en «D». Como ya se mencionó, al interior de casi todos los recintos hundidos, había un apisonado regular que se formó sobre una delgada capa de tierra compactada o directamente sobre la capa natural. Dicho apisonado presenta residuos de tierra quemada, carbones y fragmentos de cerámica dispuestos en posición horizontal, pero no parece que hayan sido utilizados como un espacio doméstico. En algunos recintos, además, se registraron pequeños hoyos y pozos excavados en la capa natural, algunos de los cuales contenían piruros o puntas de obsidiana que sugiere su uso como pozos de ofrenda. Una piedra alargada dispuesta como escalón servía para entrar y salir de estos recintos.

El segundo momento de ocupación ocurrió después de que los recintos hundidos fueron totalmente rellenados con una gruesa capa de tierra mezclada con piedras, ceniza y otros deshechos, sobre la cual se dispuso una delgada capa de tierra compactada y se formó un segundo nivel de uso que ocupaba todo el interior de los recintos en «D». Este segundo nivel de uso se encontraba casi 


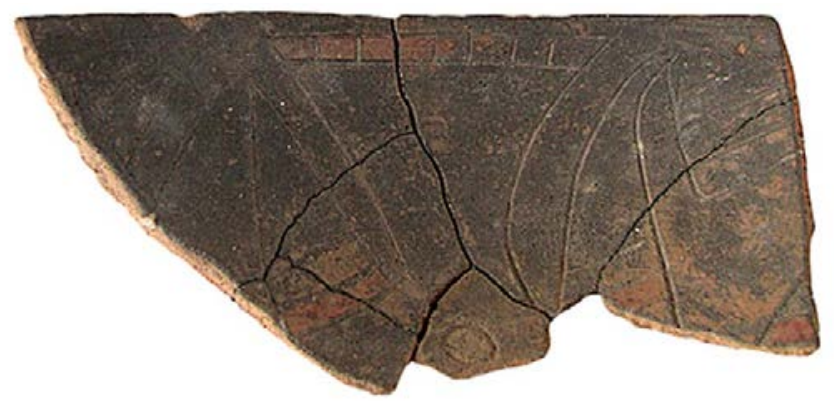

10

$\mathrm{cm}$

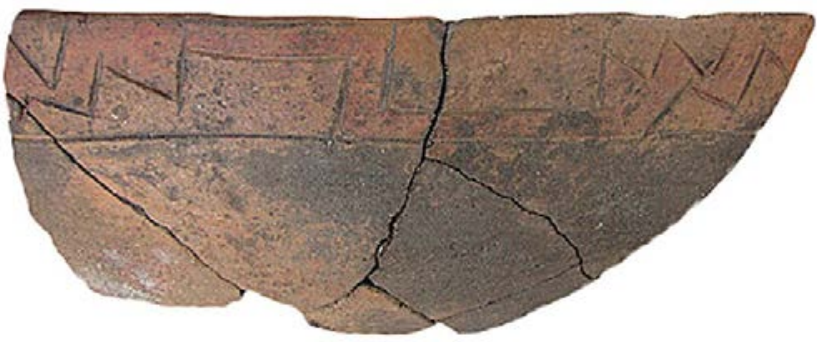

Figura 14. Fragmento de cerámica de una vasija — cuenco abierto- típica de la fase Ocucaje 9, encontrada en Cutamalla, decorada tanto al interior como al exterior con motivos geométricos y figurativos (foto: J. Isla).

en la superficie de los recintos en "D», en relación con la base de los muros, apenas cubierta por delgadas capas de tierra suelta y hierbas.

Aunque no se ha llegado a definir con certeza la diferencia temporal entre el primer y segundo momento de ocupación, los materiales asociados, especialmente los fragmentos de cerámica, indican claramente que el primer momento ocurrió durante la época Paracas Tardío, específicamente en relación con la fase Ocucaje 9 (Fig. 14), mientras que el segundo, durante la época de transición de Paracas a Nasca (incluye materiales de la fase Ocucaje 9 pero también otros fragmentos de cerámica de las fases Ocucaje 10 y Nasca 1), lo que indicaría que se trata de una ocupación sucesiva.

Los materiales arqueológicos encontrados asociados con ambos momentos de ocupación incluyen una regular cantidad de fragmentos de cerámica, mayormente cerámica no diagnóstica, pero con varios fragmentos decorados (Fig. 15a, 15a). Una vasija completa de cerámica fina decorada con líneas incisas se encontró en la UA-3, asentada en el borde noreste del recinto hundido. También, se registró un buen número de artefactos de cerámica que incluyen piruros (Fig. 16) y alisadores que se habrían utilizado para el hilado y la fabricación de la cerámica, respectivamente. Entre los artefactos líticos, destaca el hallazgo de numerosas puntas de obsidiana (Fig. 17), además de lascas y esquirlas del mismo material, lo cual nos permite inferir que las puntas se producían en estos espacios, aunque no se ha llegado a distinguir un lugar específico de talla. También, se encontraron algunas manos de moler, raspadores, chancadores y percutores de piedra, lo que sugiere el desarrollo de actividades domésticas limitadas en alguno de estos recintos. Huesos fragmentados de animales — principalmente de camélidos_ han sido hallados mayormente en relación con el segundo momento de ocupación. 


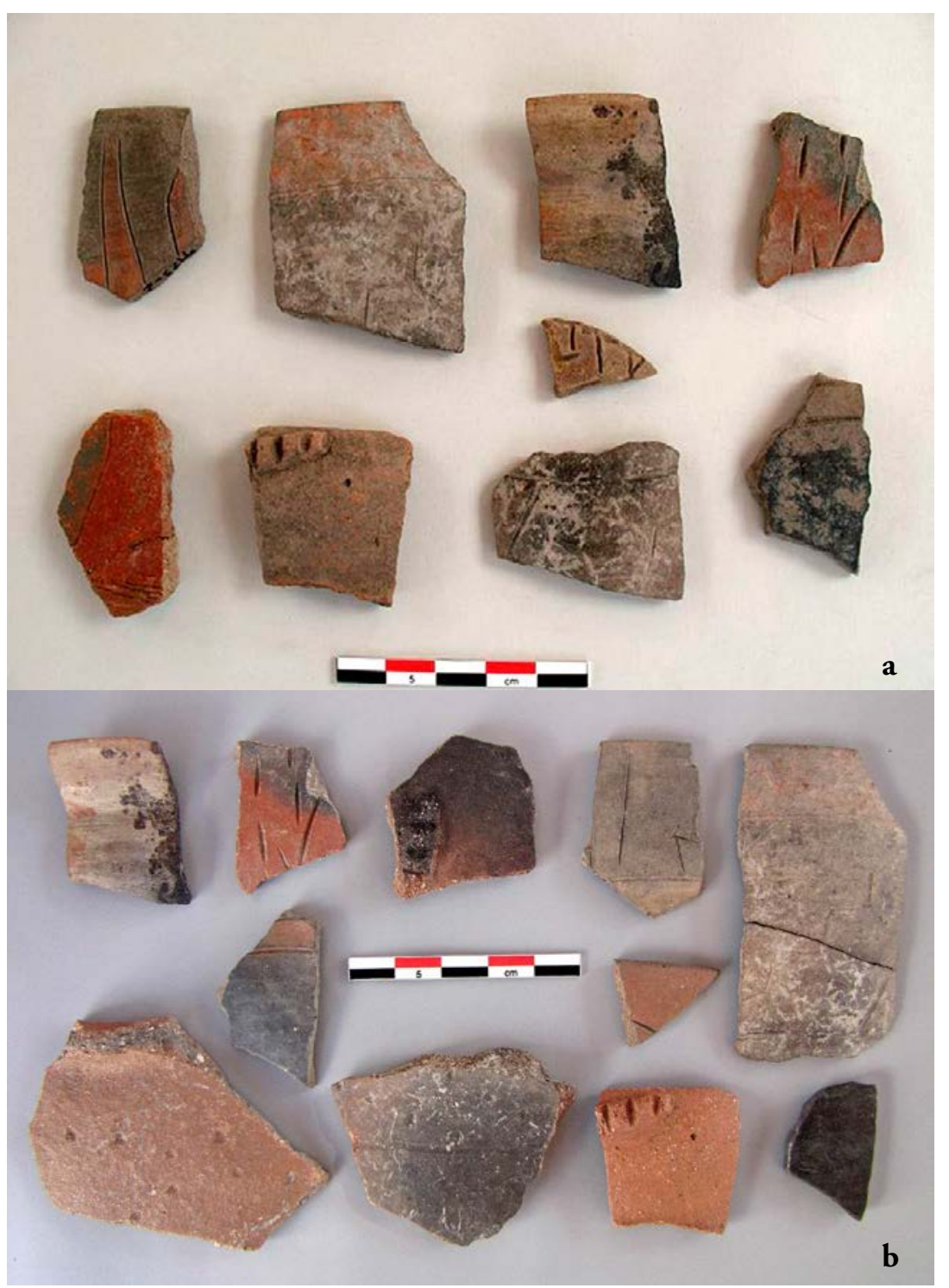

Figura 15a-b. Fragmentos de cerámica correspondientes principalmente a cuencos, ollas y cántaros encontrados en Cutamalla, en donde destacan varios fragmentos incisos y pintados (foto: J. Isla).

\subsection{Análisis y resultados}

Las excavaciones realizadas en el sector B de Cutamalla nos permitieron exponer más de la mitad de una de las estructuras arquitectónicas en forma de flor. Dicho sector se compone de un patio central hundido de 16,5 metros de diámetro, alrededor del cual, dejando un espacio inclinado libre de construcciones, se establecieron 16 recintos en forma de «D» que estaban dispuestos simétricamente uno al lado del otro con su sección recta orientada hacia el patio hundido. ${ }^{13}$ Todos los recintos en «D» incluyen una pequeña terraza delante del muro recto del recinto, orientado hacia el patio, mientras que, al interior de los recintos en «D», había un pequeño recinto hundido de contorno circular. La conformación arquitectónica de estas estructuras evoca a la forma de una flor 


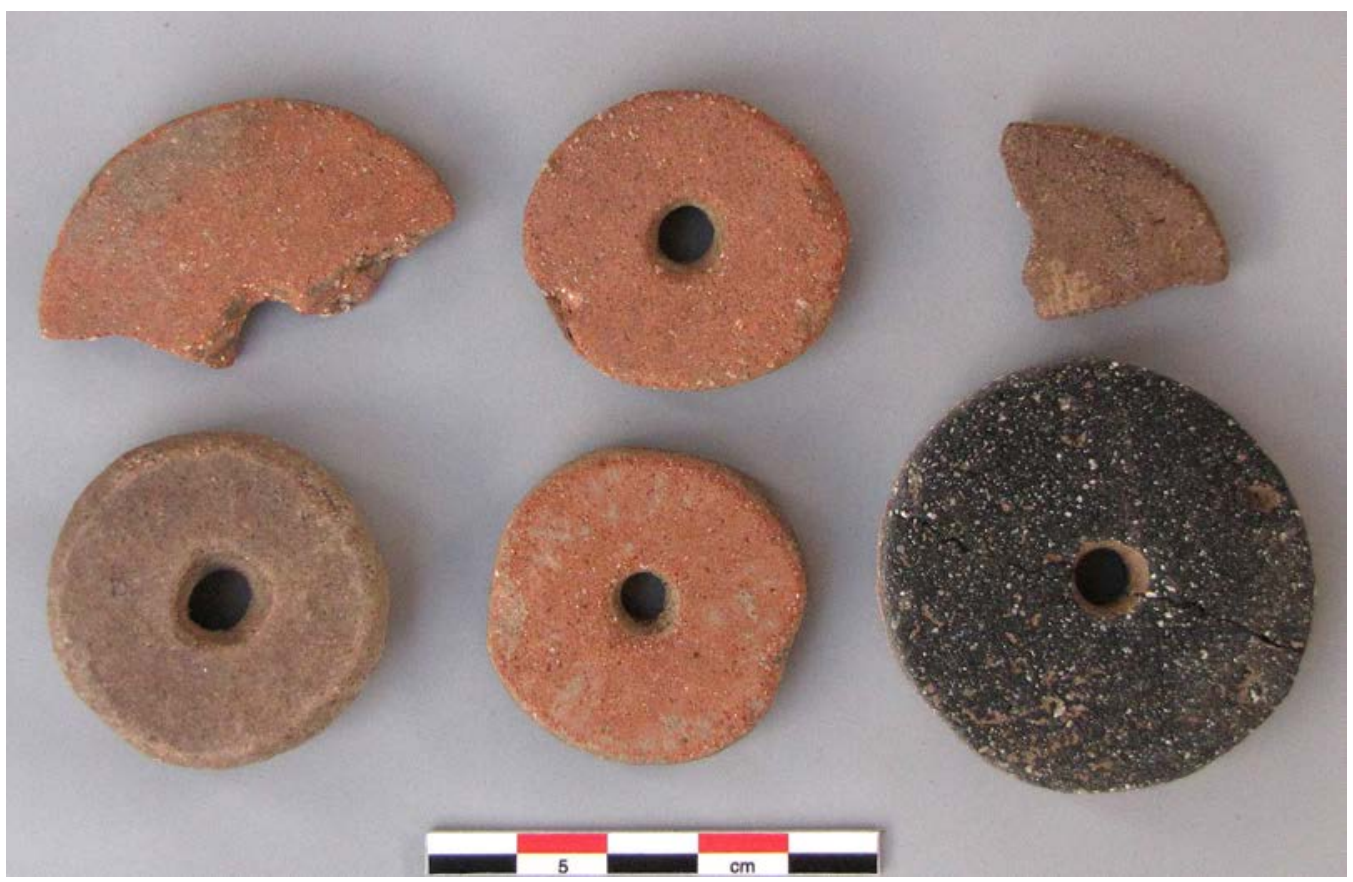

Figura 16. Muestra de los numerosos piruros de cerámica encontrados en las excavaciones y que fueron utilizados en el hilado de las fibras de camélidos (foto: J. Isla).

que hasta el momento no se ha registrado en otro lugar asociada con la cultura Paracas; de hecho, es una novedad en todos los Andes centrales.

Según los rasgos arquitectónicos observados —-materiales de construcción, técnica constructiva, forma y organización de los recintos- , se puede afirmar que cada una de las estructuras arquitectónicas en forma de flor en Cutamalla forman parte de un mismo diseño y modelo arquitectónico. En este sentido, tanto el patio hundido como los recintos en "D" y sus respectivas terrazas fueron construidos simultáneamente como parte de un proyecto previamente elaborado. La simetría y el orden que muestran estas estructuras confirman esta observación. A partir de esto, el establecimiento de un recinto hundido al interior de cada recinto en «D» fue parte del proceso constructivo que, aunque no fue igual en todos sus detalles, se puede decir que sigue un modelo único.

En este contexto, las excavaciones han permitido identificar dos momentos de ocupación bien definidos. El primero relacionado con un apisonado establecido tanto al interior de los recintos hundidos como en su entorno superior, sobre la capa natural, y el segundo, con un apisonado que se formó después de rellenar los recintos hundidos, sobre una capa de tierra que estaba en relación con la base del muro que delimita los recintos en «D». En el caso del patio hundido, el primer momento de uso se relaciona con la superficie del terreno, en el que había varios silos, algunos pozos de quema y hoyos de poste excavados en la capa natural. El segundo momento se relaciona con un piso regular que tenía una ligera inclinación hacia la parte central del patio, el cual cubría los rasgos antes indicados. Dicho piso fue afectado en su grado de dureza y compactación por la constante filtración de agua desde la superficie. ${ }^{14}$

La diferencia observada entre un momento y otro es posible que se vincule con funciones diferentes del patio en cada momento, aunque en ambos casos parece que funcionó como un espacio público abierto. En este sentido, se debe resaltar que el tamańo de las estructuras arquitectónicas excavadas y la organización de las mismas en un espacio central hundido refuerzan esta impresión, al mismo tiempo que son pocas las evidencias de contextos domésticos en las estructuras en forma de flor. 


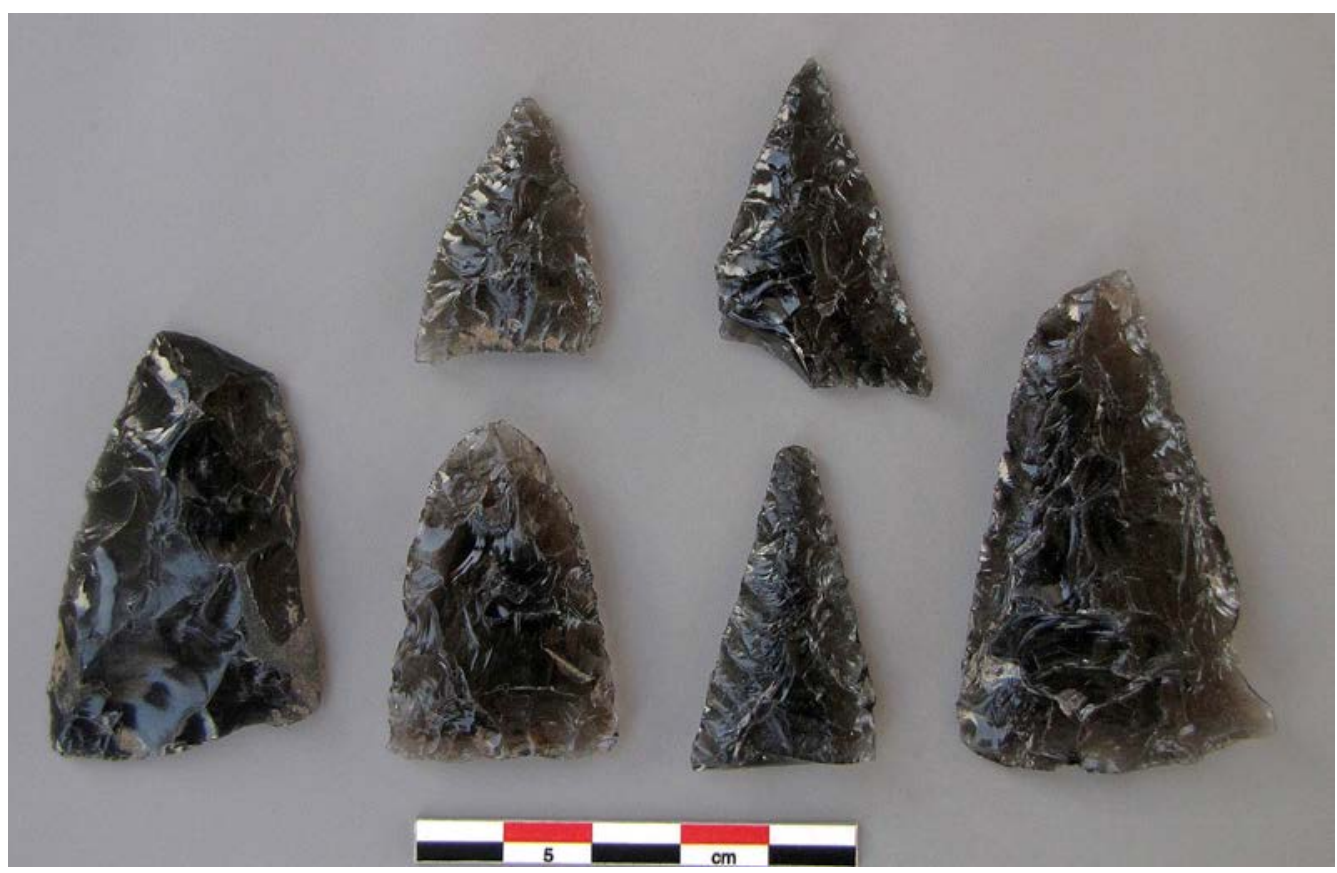

Figura 17. Ejemplo de algunas puntas y cuchillos de obsidiana finamente trabajados que se han encontrado en todas las unidades excavadas en Cutamalla (foto: J. Isla).

Según la cerámica asociada, en relación con ambos momentos de uso se han registrado materiales de la fase Ocucaje 9 (Paracas Tardío), aunque también en el segundo momento se encuentran fragmentos de la fase Ocucaje 10, correspondiente a la época de transición entre Paracas y Nasca, lo que estaría indicando el uso continuo de esos espacios durante las dos últimas fases de desarrollo de la cultura Paracas.

Desde esta perspectiva, la forma tan regular y concéntrica de las estructuras en forma de flor sugiere a priori que, en ellas, se desarrollaban actividades rituales o ceremoniales. Sin embargo, el hallazgo de muchos piruros, así como de puntas (incluidas lascas y esquirlas) de obsidiana, indican que allí se habrían realizado actividades productivas relacionadas con el procesamiento de la lana de camélidos y la fabricación de puntas de obsidiana. Como sabemos, se trata de recursos provenientes casi exclusivamente de la sierra, los cuales tenían una gran demanda en la costa sur, en particular, en la parte más tardía del desarrollo de la cultura Paracas, cuando ocurrió un auge en la producción de textiles hechos con fibras de camélidos, así como también en la necesidad de tener una mayor cantidad de puntas y cuchillos de obsidiana. El hallazgo de numerosos artefactos utilizados en la producción de cerámica indica que también esta era una actividad importante en el lugar.

Todas estas evidencias sugieren que las principales actividades realizadas en las estructuras en forma de flor se relacionan con la producción de bienes, cuya materia prima era obtenida y procesada en la sierra, y, luego, transportada hacia los sitios de la costa, especialmente hacia aquellos localizados en los valles de Ica, Pisco y Chincha, y también en la península de Paracas (Fig. 18). Así mismo, el descubrimiento de silos y de posibles depósitos tanto en el patio hundido como al interior de los recintos en «D», respectivamente, sugieren que en Cutamalla se realizaba el acopio y almacenamiento de productos de la zona que luego podían ser intercambiados con la costa. 


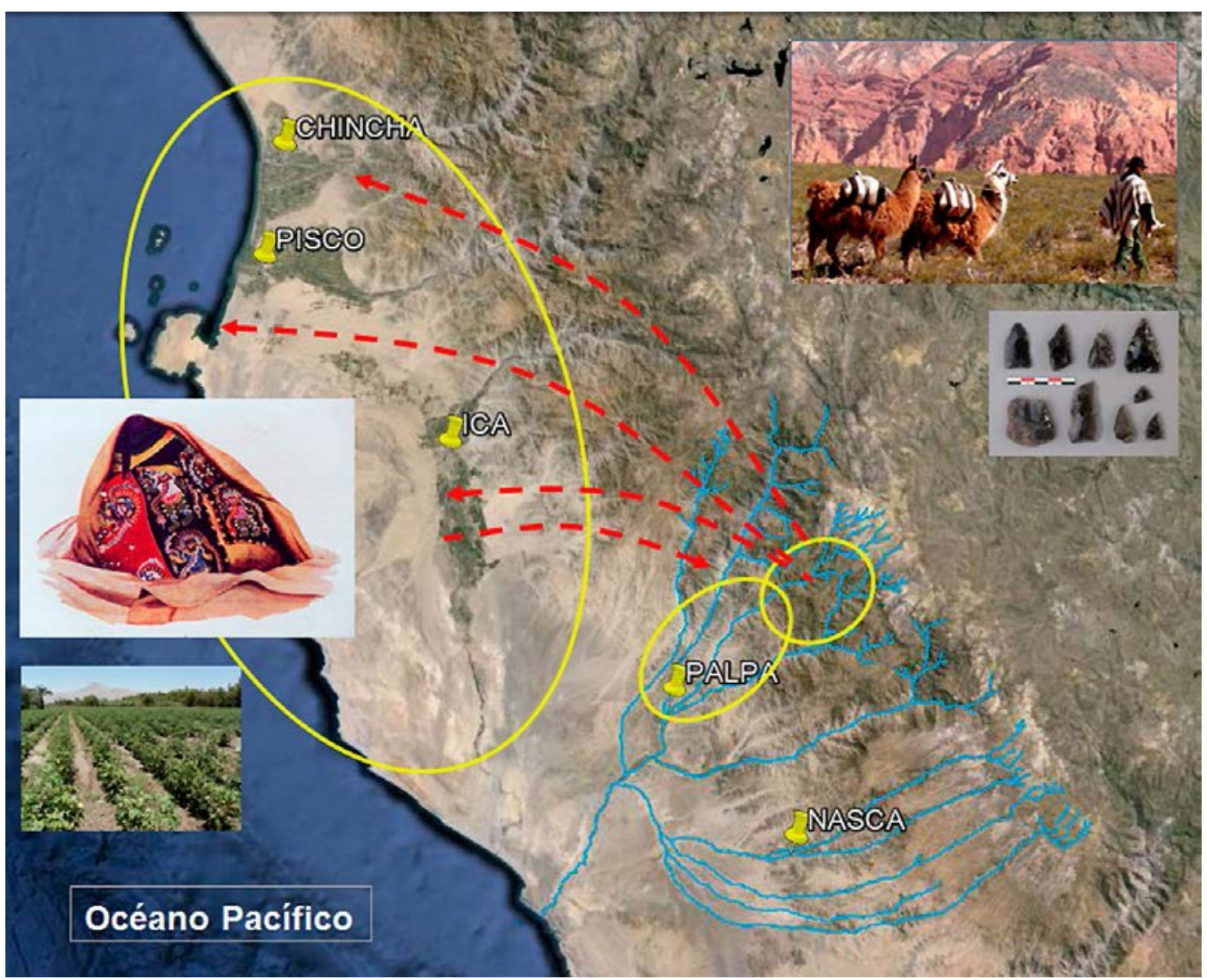

Figura 18. Mapa de la costa y sierra sur del Perú, donde se muestra la zona de estudio - en la sierra- en relación con el área nuclear de la cultura Paracas en la costa, entre las cuales habría existido una importante red de intercambio (gráfico elaborado por los autores).

\section{Sitios de la época de Transición de Paracas a Nasca}

En la zona de investigación, se han registrado pocos sitios pertenecientes a la época de transición de Paracas a Nasca (fases Ocucaje 10 y Nasca 1), pero es muy probable que varios otros sitios se encuentren cubiertos por tierra o debajo de ocupaciones posteriores. Tal es el caso de Huayuncalla, en donde se encuentra una ocupación de ese tiempo cubierta por construcciones pertenecientes a una ocupación de la cultura Wari en la zona. Un caso distinto pero relacionado con este tema es el observado en Cutamalla, en el sector A, en donde la destrucción de las estructuras arquitectónicas y la falta de materiales de superficie tampoco permitió distinguir una importante ocupación de ese tiempo.

No obstante, en ambos casos, las excavaciones han permitido descubrir estructuras arquitectónicas parecidas a aquellas documentadas en el sector B de Cutamalla, en las que se observa una aparente continuidad de las construcciones en forma de flor, pero que difieren en ciertos detalles formales y constructivos.

En el caso de Cutamalla, durante esta época, se establecieron recintos en forma de «D» similares a los del sector $\mathrm{B}$, pero un poco más grandes y que forman unidades individuales que, delante del muro recto, presentan una especie de banqueta con subdivisiones. Más adelante, se encuentra un patio bastante grande, pero que solo se encuentra en relación con el recinto en «D». Es decir, esta vez, los recintos en "D» presentan su propio patio delimitado con un muro de piedras medianas y grandes, formando unidades arquitectónicas diferentes a las estructuras en forma de flor. 
En el sector A de Cutamalla, se han excavado al menos cuatro estructuras similares. Evidentemente, se trata de un nuevo tipo de estructuras arquitectónicas que al parecer solo se limita a este sitio.

Por otro lado, en el caso de Huayuncalla, las excavaciones permitieron descubrir una ocupación de la época de transición de Paracas a Nasca, conformada por recintos en forma de «D» o de contorno ligeramente más ovalado a circular, pero muy parecidos a las estructuras arquitectónicas en forma de flor. Al parecer, estos recintos, que también tienen el pequeño recinto circular excavado en su interior, se disponen alrededor de un patio bastante grande. Cabe anotar que no se ha llegado a comprobar si es hundido o no. En todo caso, las construcciones formalmente son más irregulares y menos elaboradas que las estructuras en forma de flor. La cerámica asociada a estas construcciones se relaciona con la cultura Topará de la costa.

Finalmente, en este punto, es importante indicar que otros tres sitios (PAP- 1077, 1082 y 1083) con características similares a las de Cutamalla se han registrado la zona yunga, en el sector de Collanco, en la parte alta del valle de Palpa. Se trata de sitios que datan igualmente de la época de transición de Paracas a Nasca, en los que también se observan recintos en forma de «D» dispuestos alrededor de patios que forman varios grupos o unidades arquitectónicas. En estos casos, los patios presentan un contorno más ovalado e irregular, mayormente adaptados a la topografía del terreno y en ningún caso se han registrado patios hundidos.

Las evidencias registradas en estos sitios indican que las actividades que se realizaban en estas estructuras arquitectónicas estaban relacionadas con la producción de bienes; es decir, eran similares a las que se cumplían en las estructuras en forma de flor de Cutamalla, aunque también se habrían desarrollado actividades domésticas. En todo caso, es necesario realizar mayores excavaciones en estos sitios para definir con mayor certeza las funciones que tenían.

\section{Resumen y comentarios finales}

Las prospecciones arqueológicas en la parte alta de los valles de Palpa, en la zona de «Las Cabezadas», han permitido registrar una importante ocupación prehispánica perteneciente casi a todos los períodos de tiempo. Salvo la ocupación wari durante el Horizonte Medio e Inka durante el Horizonte Tardío, las demás ocupaciones en la zona ocurrieron en relación con culturas tradicionalmente consideradas costeńas, como son Paracas, Nasca e Ica-Chincha. La presencia de estas culturas en la sierra, arriba de los 2500 a 3000 metros de altura, indica que en esta parte de la vertiente occidental de los Andes la costa y la sierra estaban integradas desde épocas muy tempranas, incluso desde el período Arcaico (Reindel e Isla 2013). Al respecto, basta con recordar que esta zona se encuentra justo en la ruta que conduce directamente hacia los ricos yacimientos de obsidiana localizados en Quispisisa, Ayacucho (Burger y Glascock 2000; Tripcevich y Contreras 2011), desde donde dicha materia prima era transportada hacia la costa y otras regiones del Perú. ${ }^{15}$

En este contexto, las primeras evidencias de asentamientos estables identificadas hasta el momento en la zona datan recién del Horizonte Temprano, aunque posiblemente todavía se encuentren ocupaciones más tempranas que no se han podido registrar con trabajos de superficie. Así, al margen de algunos sitios de la época Paracas Temprano, los lugares de asentamiento más seguros pertenecen a las épocas Paracas Medio y Paracas Tardío, cuando la zona fue ocupada de manera más estable y permanente.

Por otro lado, los materiales asociados — principalmente fragmentos de cerámica — indican que las estructuras arquitectónicas en forma de flor datan principalmente de la época Paracas Tardío (480 a 200 a.C.), relacionadas principalmente con la fase Ocucaje 9, aunque en el sitio de Quechqalla (PAP-770) también se han registrado materiales de la fase Ocucaje 8. Esto, en todo caso, indica de algún modo que este tipo de estructuras arquitectónicas aparecieron en relación con la fase de reorganización que experimentó la cultura Paracas en la última etapa de su desarrollo (Massey 1986, 1991; DeLeonardis 1997; Peters 1997; Bachir Bacha y Llanos 2013).

Hasta el momento, las evidencias documentadas en la zona de estudio indican que se trata de un modelo arquitectónico único que se encuentra restringido solo a una parte de la sierra de Lucanas, 
específicamente a la zona comprendida entre los distritos de Laramate y Ocaña, en la vertiente occidental de los Andes. Al parecer, se trata de un modelo arquitectónico local que, hasta el momento, no tiene comparación con otros lugares de la zona, como, por ejemplo, en las nacientes de los ríos Ica o Nasca. Tampoco, se conocen ejemplos de otras regiones salvo algunas pocas - muy pocassimilitudes con sitios lejanos del altiplano como Chiripa (Bandy 2006) o Khonkho Wankane (Scott 2013). En todo caso, se espera en el futuro encontrar evidencias comparables en otras zonas, especialmente en la sierra sur del área andina.

En cuanto a su conformación arquitectónica, las estructuras registradas en Cutamalla muestran una composición básica: un espacio casi plano adaptado al terreno natural, en donde los recintos en «D», en número variable entre cuatro y 16, se disponen alrededor de un espacio abierto en forma de media luna, o, en el mejor de los casos, alrededor de un patio hundido. En todos los casos, los muros que conforman los recintos en «D» son bajos pero bastante sólidos, usualmente, hechos con grandes piedras dispuestas en una sola hilera y con solo una o dos hiladas. Por otro lado, los muros que delimitan los patios hundidos están conformados por lajas alargadas colocadas en forma vertical con sus caras planas hacia el exterior. Vistas en conjunto, la forma y las dimensiones de las estructuras arquitectónicas excavadas sugieren que se trata de construcciones monumentales, las cuales deben ser entendidas en un contexto local y considerando que la palabra grande no lo expresa en su verdadera dimensión.

Las excavaciones en el sector B de Cutamalla han permitido descubrir más de la mitad de una de las estructuras arquitectónicas en forma de flor, la más completa de todas, la cual presenta un patio hundido de contorno circular, alrededor del cual y de manera simétrica se establecieron 16 recintos en forma de «D» con sus respectivas terrazas. Los rasgos identificados y los materiales encontrados tanto en el patio hundido como en los recintos en «D» nos han permitido identificar claramente dos momentos de ocupación. El primero se relaciona con la construcción y uso de las construcciones durante la última fase de la época Paracas Tardío, es decir, en la fase Ocucaje 9. El segundo corresponde a la época de transición de Paracas a Nasca (Ocucaje 10 y Nasca 1), cuando se reutilizaron algunos recintos en «D» después de que sus estructuras interiores habían sido rellenadas y abandonadas. ${ }^{16}$

Teniendo en cuenta los rasgos formales y constructivos, así como los materiales asociados, se puede afirmar con bastante certeza que las estructuras arquitectónicas en forma de flor corresponden a construcciones de carácter público, en donde se habrían realizaron principalmente actividades relacionadas con el procesamiento de bienes. Aunque las evidencias recuperadas no son del todo concluyentes, es evidente que el patio hundido — rodeado de los recintos en «D»— tuvo una función especial: se constituyó como el espacio en donde posiblemente también se realizaron actividades festivas y religiosas. Desde esta perspectiva, se debe recordar que en el mundo andino ambas funciones - producción de bienes, festividad y/o religiosidad - no son excluyentes, sino más bien complementarias, situación que evidentemente también alcanzó a los habitantes de la cultura Paracas en la sierra.

En este sentido, es posible que, durante la época Paracas Tardío, Cutamalla y los otros sitios registrados en la zona hayan sido los centros principales de una colonia paracas conformada por una pequeña élite; grupos de artesanos; $y$, principalmente, por agricultores y pastores que, respectivamente, cultivaban las numerosas terrazas agrícolas dispersas en la zona y realizaban el acopio de lana de camélidos en los numerosos rebańos dispersos en las alturas. Los artesanos, por su parte, se habrían dedicado a la fabricación de puntas y cuchillos de obsidiana, cuya materia prima procedía de los yacimientos de obsidiana de Quispisisa localizados al otro lado de la meseta andina, en las alturas de Huancasancos, Lucanas, mientras que otro grupo de artesanos alfareros se dedicaban a la elaboración de cerámica, mayormente de tipo utilitario, para el consumo local.

En este punto, es importante indicar que el acopio y el procesamiento de la lana de camélidos debieron haber sido unas de las actividades más importantes en la zona, puesto que justamente en este tiempo (Ocucaje 9) ocurrió la mayor demanda de materias primas para la fabricación de textiles en la costa, donde — como todos sabemos — se dio un auge en la producción de textiles. 
Esta actividad parece haberse intensificado durante la época de transición de Paracas a Nasca, correspondiente a la fase Necrópolis (Ocucaje 10 y Nasca 1), en relación con la producción de textiles para los fardos funerarios descubiertos en la península de Paracas y otros sitios del valle de Ica, los cuales se relacionan con la cultura Topará (Wallace 1986; Peters 1997). Seguramente, esta red de intercambio también alcanzó al valle de Chincha, donde se encuentran varios sitios importantes de las culturas Paracas y Topará (Canziani 1992; Tantaleán et al. 2013).

Teniendo en cuenta que los centros poblados más importantes de la cultura Paracas estaban en la costa, en los valles de Chincha, Pisco e Ica, se entiende que en la sierra se procesaban diversos productos que fueren almacenados temporalmente en depósitos y, luego, transportados en caravanas de llamas hacia la costa. $\mathrm{Al}$ respecto, las fuentes etnohistóricas nos informan que, desde la costa, en cambio, se transportaron algodón, ají y otros productos agrícolas hacia la sierra (Guillén 1984).

\section{Agradecimientos}

Queremos agradecer al Ministerio Federal de Educación e Investigación de Alemania por el financiamiento brindado parra las investigaciones del Programa de Investigaciones denominado "Transecta Andina», en el marco del cual se desarrollaron los proyectos arqueológicos Nasca-Palpa y Palpa-Lucanas. Dicho programa tenía como objetivo estudiar el desarrollo cultural y los sistemas económicos de las poblaciones prehispánicas establecidos en los valles de Palpa. Al mismo tiempo, agradecemos al Instituto Nacional de Cultura (hoy Ministerio de Cultura) por habernos brindado los permisos y las facilidades correspondientes para realizar nuestras investigaciones.

También, va nuestra gratitud a los arqueólogos peruanos y alemanes (del Instituto Arqueológico Alemán) que participaron en los trabajos de prospección y excavación en la zona, así como a las autoridades locales y a todo el personal técnico (obreros) que nos apoyaron en las labores de campo y gabinete.

\section{Notas}

${ }^{1}$ La parte alta de los valles de Palpa, más conocida como «Las Cabezadas», políticamente se localiza en la provincia de Lucanas, región de Ayacucho.

${ }^{2}$ En la actualidad, debido al cambio climático y al incremento de ganado caprino, cada vez, es más notoria la falta de lluvias y la escasez de pastos, lo cual está dejando grandes extensiones de lomas y laderas carentes de vegetación.

${ }^{3}$ Las investigaciones en la zona de la sierra se realizaron en el marco de los Proyectos Arqueológicos Nasca-Palpa y Palpa-Lucanas, dirigidos por los autores de este artículo. Dichas investigaciones formaron parte del Programa de Investigaciones denominado "Transecta Andina», el cual fue financiado por el Ministerio Federal de Educación e Investigación de Alemania.

${ }^{4}$ Los trabajos de prospección estuvieron a cargo de Carolina Hohmman y Johny Isla, quienes cada año contaron con el apoyo y asistencia de varios arqueólogos y estudiantes del Perú y Alemania.

${ }^{5}$ En los estudios en la sierra, hemos utilizado el mismo esquema cronológico de la costa debido a la gran similitud que muestran los materiales registrados en el campo (Rowe 1960; Menzel et al. 1964; Menzel 1964, 1976; Hecht 2013).

${ }^{6}$ Teniendo en cuenta la directa asociación de estas terrazas con sitios de ocupación paracas (p.e. Cutamalla, Collanco, Qechcalla, etc.), se puede afirmar que la mayoría de las terrazas agrícolas datan del tiempo de la ocupación paracas en la zona, las cuales luego fueron reutilizadas por otras poblaciones a lo largo del tiempo. En el caso de Cutamalla, si bien las terrazas no han sido fechadas, los únicos materiales encontrados en relación con ellas datan de la época Paracas Tardío, al igual que en Collanco. 
${ }^{7}$ Debido a que la ganadería es la base económica de los pobladores de la zona, en la actualidad, solo una pequeña parte de las antiguas terrazas de cultivo todavía siguen en uso.

${ }^{8}$ En la zona de estudio, se identificó un camino de larga distancia (con ramales) que transcurre por la cima de una cadena de cerros que conforma la divisoria de aguas entre los valles de Llauta y Laramate.

${ }^{9}$ En Mallma, como en otros sitios bastante grandes, además de la ocupación paracas, también se han encontrado materiales pertenecientes a otros períodos de tiempo, lo que indica que algunas partes del sitio fueron reutilizados u ocupados en otras épocas.

${ }^{10}$ Solo tres de estas estructuras están completas; es decir, tienen el patio hundido y recintos en «D» en su alrededor. Las demás están incompletas: tienen forma de media luna, y solo presentan parte del patio y algunos recintos en «D».

${ }^{11}$ Las demás estructuras arquitectónicas varían solamente en algunos aspectos como el tamaño, número de recintos en «D», detalles constructivos menores y estado de conservación.

${ }^{12}$ Algunas piedras o lajas son bastante grandes y alargadas, por lo que el muro en algunas partes es visible desde la superficie, aunque su mayor parte se descubrió recién después de retirar las primeras dos capas.

${ }^{13}$ En total, las excavaciones en el sector B cubrieron un área de casi de 800 metros cuadrados distribuidos en ocho unidades de diez por diez metros de lado, las cuales estaban ubicadas una al lado de la otra siguiendo el sistema de coordenadas previamente definido para el sitio.

${ }^{14}$ Teniendo en cuenta estos aspectos, es posible que, al tratarse de un mismo diseño arquitectónico, el piso del patio hundido haya sido renovado constantemente y utilizado en relación con ambos momentos de ocupación.

${ }^{15}$ En el abrigo de Llamocca, localizado en la puna arriba de Laramate, se registraron artefactos de obsidiana pertenecientes a una ocupación del período de cazadores y recolectores, cuyo fechado de radiocarbono data de hace 8000 años a.C. (Reindel 2012).

${ }^{16}$ Cronológicamente, esto concuerda bastante bien con la situación observada en los valles de la costa, desde Chincha (Canziani 2009, Tantaleán et al. 2013, Stanish et al. 2014) hasta Palpa/Nasca (Isla y Reindel 2017, e.p.), donde ambos momentos están bien representados, y se relacionan con las culturas Paracas y Topará, respectivamente.

\section{REFERENCIAS}

\section{Bachir Bacha, A. y D. Llanos}

2013 ¿Hacia un urbanismo Paracas en Ánimas Altas/Ánimas Bajas (valle de Ica)?, en: A. Bachir Bacha y J. Dulanto (eds.), Paracas: nuevas evidencias, nuevas perspectivas, Boletín de Arqueología PUCP 17, 169204.

Bandy, M.

2006 Early village society in the Formative Period in the southern Lake Titicaca Basin, en: W. H. Isbell y H. Silverman (eds.), Andean Archaeology III: North and South, 210-236, Springer, New York. https://doi. org/10.1007/0-387-28940-2_10

Burger, R. y M. D. Glascock

2000 Locating the Quispisisa obsidian source in the Department of Ayacucho, Peru, Latin American Antiquity 11 (3), 258-268. https://doi.org/10.2307/972177

Canziani, J.

1992 Arquitectura y urbanismo del Período Paracas en el valle de Chincha, Gaceta Arqueológica Andina 22, 87-117, Instituto Andino de Estudios Arqueológicos (Indea), Lima. 
2009 Ciudad y territorio en los Andes: contribuciones a la historia del urbanismo prehispánico, Fondo Editorial de la Universidad Católica del Perú, Lima.

\section{DeLeonardis, L.}

1997 Paracas settlement in Callango, lower Ica Valley, 1st millennium B.C., Peru, tesis de doctorado, Department of Anthropology, School of Arts and Sciences of the Catholic University of America, Washington, D.C.

Guillén G., E.

1984 Las oarcialidades de Hatun Rukana y Laramati en el siglo XVI: Represión de la campaña anticristiana de 1569 y descripción de sus pueblos en 1586, Boletín de Lima 33 (6), 71-82, Editorial Los Pinos, Lima.

Hecht, N.

2013 A relative sequence of Nasca style pottery from Palpa, Peru, tesis de doctorado, Facultad de Artes y Humanidades de la Universidad de Bonn, Alemania, Édition Linguistique VVB Laufersweiler Verlag.

Isla C. J.

2009 From hunters to regional lords: funerary practices in Palpa, Peru, en: M. Reindel y G. Wagner (eds.), New technologies for archaeology, 119-139, Natural Science in Archaeology. Springer-Verlag Berlín Heildelberg. https://doi.org/10.1007/978-3-540-87438-6_8

2010 Perspectivas sobre el proceso cultural en los valles de Palpa, costa sur del Perú, en: L. Valle A. (ed.), Arqueologia y desarrollo. Experiencias y posibilidades en el Perú, 15-52, Ediciones SIAN, Trujillo.

Isla, C. J. y M. Reindel

2008 Los Paracas del sur. Una perspectiva desde los valles de Palpa, en: E. León (ed.), Hilos del pasado. Un aporte francés al legado Paracas, 79-91, Instituto Nacional de Cultura, Lima.

2017 Palpa and Lucanas: Cultural Development Under Changing Climatic Conditions on the Western Slope of the Andes in Southern Peru, en: Allen Casey (ed.), The Andes: Geography, Diversity, and Sociocultural Impacts, Nova Science (en prensa).

Massey, S.

1986 Sociopolitical change in the upper Ica Valley, B.C. 400 to 400 A.D.: regional states on the south coast of Peru, tesis doctorado, Department of Archaeology, University of California, Los Angeles.

1991 Social and political leadership in the lower Ica Valley: Ocucaje phases 8 and 9, en: A. Paul (ed.), Paracas art and architecture: object and context in south coastal Peru, 315-348, University of Iowa Press, Iowa City.

Menzel, D.

1964 Style and time in the Middle Horizon, Nawpa Pacha 2, 1-105, Institute of Andean Studies, Berkeley.

1976 Pottery Ssyle and society in Ancient Peru, University of California Press, Berkeley.

Menzel, D., J. H. Rowe y L. Dawson

1964 The Paracas pottery of Ica: a study in style and time, University of California Publications in American Archaeology and Ethnology 50, University of California Press, Berkeley.

Peters, A. H.

1997 Paracas, Topará and Early Nasca: ethnicity and society on the south central Andean coast, tesis de doctorado, Department of Anthopology, Cornell University Ithaca, Ithaca.

Reindel, $M$.

2009 Life at the edge of the desert - Archaeological reconstruction of the settlement history in the valleys of Palpa, Peru, en: M. Reindel y G. Wagner (eds.), New technologies for archaeology, 439-461, Natural Science in Archaeology, Springer, Verlag Berlín Heildelberg.

2010 Archäologische forschungen der Jahre 2007 und 2008 im Anden-Transekt, Süd-Peru, Zeitschrift für Archäologie Außereuropäischer Kulturen 3, 207-224.

2012 Archäologische forschungen der Jahre 2009 und 2010 im Anden-Transekt, Süd-Peru, Zeitschrift für Archäologie Außereuropäischer Kulturen 4, 370-384.

Reindel, M. y J. Isla

2013 Cambio climático y patrones de asentamiento en la vertiente occidental de los Andes del sur del Perú, Diálogo Andino 41, 83-99. https://doi.org/10.4067/S0719-26812013000100006

2016 Nuevos estudios sobre la cultura Paracas en la vertiente occidental de los Andes, costa y sierra sur del Perú, ms. manuscrito inédito, Comisión de Culturas no Europeas/Instituto Arqueológico Alemán, Bonn. 
Reindel, M. y G. A. Wagner

2009 New technologies for srchaeology. Multidisciplinary investigations in Palpa and Nasca, Peru (edición de M. Reindel y G. A. Wagner), Natural Science in Archaeology, Springer-Verlag Berlín Heildelberg. https:// doi.org/10.1007/978-3-540-87438-6

Rowe, J. H.

1960 Nuevos datos relativos a la cronología del estilo Nasca, en: R. Matos (ed.), Antiguo Perú: espacio y tiempo, 29-45, Editorial Juan Mejía Baca, Lima.

Smith, Scott C.

2013 Late Formative Period spatial organization at KhonKho Wankane, Bolivia, en: A. Vranich y A. R. Levine (eds.), Advances in Titicaca Basin archaeology 2, 23-44, Los Angeles.

Stanish C., H. Tantaleán, B. Nigra y L. Griffin

2014 A 2300 years-old architectural and astronomical complex in the Chincha Valley, Peru, Proceedings of the National Academic of Science 111 (20), 7218-7223. https://doi.org/10.1073/pnas.1406501111

Tantaleán, H., C. Stanish, M. Zegarra, K. Pérez, B. Nigra

2013 Paracas en el valle de Chincha: nuevos datos y explicaciones, en: A. Bachir Bacha y J. Dulanto (eds.), Paracas: nuevas evidencias, nuevas perspectivas, Boletín de Arqueología PUCP 17, 31-56.

Tripcevich, N. y D. A. Contreras

2011 Quarrying evidence at the Quispisisa obsidian source, Ayacucho, Peru, Latin American Antiquity 22, 121-136. https://doi.org/10.7183/1045-6635.22.1.121

Wallace, D.

1986 The Topará Tradition: an overview, en: D. H. Sandweiss y D. P. Kvietok (eds.), Perspectives on Andean prehistory and protohistory, 35-47, Cornell University Latin American Studies Program, Ithaca.

Recepción: marzo de 2017 Aceptación: mayo de 2017 